\title{
Valuation of the Vulnerable Option Price Based on Mixed Fractional Brownian Motion
}

\author{
Yanmin Ouyang $\mathbb{D},{ }^{1}$ Jingyuan Yang $\mathbb{D},{ }^{2}$ and Shengwu Zhou $\mathbb{D}^{1}$ \\ ${ }^{1}$ School of Mathematics, China University of Mining and Technology, Xuzhou, 221116, China \\ ${ }^{2}$ School of Management, China University of Mining and Technology, Xuzhou, 221116, China \\ Correspondence should be addressed to Jingyuan Yang; 130635007@qq.com and Shengwu Zhou; zswcumt@163.com
}

Received 5 April 2018; Revised 1 October 2018; Accepted 15 November 2018; Published 3 December 2018

Academic Editor: Guang Zhang

Copyright (c) 2018 Yanmin Ouyang et al. This is an open access article distributed under the Creative Commons Attribution License, which permits unrestricted use, distribution, and reproduction in any medium, provided the original work is properly cited.

The pricing problem of a kind of European vulnerable option was studied. The mixed fractional Brownian motion and the jump process were used to characterize the evolution of stock prices. The closed-form solution to European option pricing was obtained by applying martingale measure transformation method. At the end of this paper, some numerical experiments were adopted to compare the new pricing formula introduced in this paper with the classical Black-Scholes pricing formula. The result showed that the new pricing formula conformed to the actual financial market. In fact, the option value is positively correlated with the underlying asset price and the company's asset price and the jump process has significant influence on the value of option.

\section{Introduction}

Vulnerable European option is a kind of option with credit risk, which refers to the possibility of one party suffering losses as a result of the other party's default on the contract. The pricing of credit risk has been concerned by people very early. Merton (1974) [1] introduced option pricing theory into zero coupon bond credit risk pricing at first time. He supposed that the capital structure of company consists of asset and debt. A default occurred when the company becomes insolvent at the expiration of the option. In this model, a baseline of structured-form model ideas was built. Later, the irrational assumption was improved in Merton's model and the first passage time model was proposed by Black and Cox (1976)[2]. Johnson and Stulz (1987) [3] firstly led default risk to option pricing and put forward a new conception called vulnerable option. Klein (1996) [4] obtained the pricing formula of vulnerable option by the method of martingale. The default risk was decomposed into timing and recovery risks by Madan (1998) [5] who generated estimation strategies evaluating the recovery risk and then constructed implicit prices of contingent securities reflecting the timing risk. Lobo (1999) [6] and Zhou (2001) [7] considered the pricing problem of European option with the credit risk that the underlying asset price carried jump, but there were no analytical solutions in the end. Klein's option pricing model was expanded by Ammann (2001)[8] by applying structured method. They derived the closed-form solution to vulnerable option price in the environment where interest rate and default intensity complying the stochastic differential equation. In addition, Chang and Huang (2006) [9] also dealt with the problem. In the case of the underlying interest rates following a mean reverting square-root process, the analytical solutions to defaultable bonds were derived by Cathcart (2003) [10]. The reduced-form model was dated back to Jarrow and Turnbull (1995) [11]. Assuming that the default event obeyed the independent homogeneous Poisson process, the discrete methods were used to establish a simplified credit risk model by Jarrow and Turnbull. Cathcart (2006) [12] derived the price of the defaultable bond and proposed a valuation model that combined features of both the structured-form and reduced-form approaches for modelling default risk. Later, the problem that a new model of credit risk was proposed combining structural information with the reduced-form approach also was studied by Ballestra (2014) [13] and a closed-form approximate solution was derived by perturbation approach and the Laplace transform. A quasianalytical approximation of the survival probability was derived based on Madan's credit risk model, and a quasianalytical approximate expression for CDS par spreads was obtained by 
Ballestra (2017) [14]. All the discussions above are based on the underlying asset price obeying the geometric Brownian motion, which implies that the changes of underlying asset price are mutually independent random variables and the return on assets obeys the normal distribution. But in recent years many financial empirical studies showed that the distribution of return on assets had the feature of high peaks and heavy tails. Changes in stock price showed different degrees of long-term correlation and autocorrelation at different time. These characteristics are different from standard Brownian motion but similar to fractional Brownian motion. All of these features make fractional Brownian motion more widely used in assets pricing. Sottinen and Valkeila (2001) [15] proposed that there is no equivalent martingale measure in the fractional Black-Scholes market. Yaozhou $\mathrm{Hu}$ and øksendal (2003) [16] studied the structural model of credit risk under fractional Brownian motion and proved that the Black-Scholes market driven by fractional Brownian motion is a nonarbitrage market. More studies about fractional Brownian motion are introduced by øksendal (2004) [17]. However, there were some shortcomings when Fractional Brownian motion is utilized to characterize the volatility of asset price by Björk and Hult (2005) [18]. In addition, there is a main problem that fractional Brownian motion is not a semi-martingale when it is applied in financial market. In order to avoid these problems and consider the long memory characteristics of the financial asset price process, it is reasonable to use mixed fractional Brownian motion to characterize the volatility of financial assets. Mixed fractional Brownian motion is a Gaussian process and it is a linear combination of Brownian motion and fractional Brownian motion. He is Cheridito (2001) [19] who initially put mixed fractional Brownian motion of economics into use. Androshchuk (2006) [20] proved that the mixed Brownian-fractionalBrownian model had no arbitrage opportunity in the class of self-financing Markov-type strategies. A European call option pricing formula was derived in mixed Brownianfractional-Brownian environment with a mean self-financing delta hedging argument in a discrete-time setting and the minimal pricing of European option under transaction costs was obtained by Wang, Zhu and Tang (2010) [21]. Ballestra (2016) [22] studied the pricing barriier options by solving an initial-boundary value partial differential problem, in which an ad-hoc numerical procedure was employed based on product integration. Besides, Necula (2002)[23] introduced the Black-Scholes pricing formula by using quasi-martingale method in the framework of risk neutral measure, which is a generalization of the classical Black-Scholes formula. Wenli Huang (2015) [24] considered the general European option pricing model with stochastic interest rate driven by fractional Brownian motion and partial differential equation. Xiaonan Su (2012) [25] supposed that the intensity of default is driven by a jump diffusion process and got vulnerable option pricing formula in a reduced-form by using martingale method. Based on Xiaonan Su, Jinzhi Li (2013) [26] used the change of measure to obtain the closed-form solution of vulnerable option pricing. Assuming that the stock price obeyed the jump-diffusion model, Chao Wang (2015) [27] got analytic solution to vulnerable European option based on
Xiaonan Su. Adopting the Ito's formula of mixed fractional Brownian motion, Zhiguang Li (2016) [28] obtained the European option pricing formula with short-term interest rate obeying Vasicek model.

In this paper, we will mainly study the pricing problem of a class European vulnerable option under a mixed fractional Brownian motion environment. The paper is organized as follows: In Section 2, we will give pricing formula of structuredform and reduced-form model respectively. Then we will obtain the pricing formula of vulnerable option by combing the two models into Hybrid model when the stock price obeys mixed fractional Brownian motion under the jump-diffusion model. In Section 3, the analytic solution is obtained by the pricing formula. In Section 4, in order to investigate the impact of various parameters on option pricing, we give the figures of option value under different parameters with respect to the underlying asset price changes. In addition, for the reader's convenience, the last part of this paper is an appendix to the concrete proof process of vulnerable option pricing.

\section{Hybrid Model}

Merton (1974) [1] first introduced the structured-form model, which is a default model based on specific information of the company. He thought that default was related to the company's own business condition. The structured-form model considered the company's asset as underlying asset, the value of creditor's rights as the strike price, and used the option pricing method to consider default. In Merton's model, he assumed that there would be no default when the option expired, but this obviously did not match the actual financial market. Later, Black and Cox (1976) [2] improved the unreasonable assumption in Merton's model and proposed the first passage time model. The model set a boundary for company's asset and when the value of the asset fell below this boundary, a default happened. Under risk-neutral probability measure, it is assumed that the firm's value process $V_{t}$ satisfies the stochastic differential equation

$$
d V_{t}=r V_{t} d t+\sigma V_{t} d W(t)+\sigma^{H} V_{t} d W^{H}(t),
$$

where $r$ is risk-free interest rate, $W(t)$ is standard Brownian motion on probability space $\left(\Omega, \mathscr{F},\left(\mathscr{F}_{t}\right)_{0 \leq t \leq T}, P\right)$, and $W^{H}(t)$ is fractional Brownian motion whose Hurst parameter is $H(1 / 2 \leq H<1)$ on this space. $\sigma$ is the stock price volatility driven by standard Brownian motion and $\sigma^{H}$ is volatility driven by fractional Brownian motion.

It is supposed that there exists a default threshold $D>0$. When $V_{t} \leq D$, the company will default, and on the contrary, there will be no default. Hence the default time $\tau$ is given by

$$
\tau=\inf \left\{V_{t} \leq D\right\},
$$

Klein (1996)[4] assumed that if a default happened, the option seller will only pay part of the value of the equity on maturity and we denoted this ratio by $\omega$

$$
\omega=\frac{1-w}{D} V_{T}
$$


where the percentage of the cost of the bankruptcy to the option seller's asset is $w$.

The reduced-form model can trace back to Jarrow and Turnbull (1995) [11]. Unlike the structured-form model, it did not consider the relationship between the default and asset value. It regarded the process of default as a jump process. The model treated the default event as a kind of jump and often portrayed the default as a Poisson process with intensity $h$. The probability of nondefault at the moment $t$ is $e^{-h t}$ and $h$ is defined as the default density. Later Lando (1998) [29] introduced Cox process and supposed that the default process was characterized by a Cox process. The default time was the first time that Cox process jumped. This model assumed that $N$ is a Cox process and its random intensity process $\{\lambda(t): 0 \leq t \leq T\}$ satisfied

$$
\begin{aligned}
P & \left(N_{T}-N_{t}=n\right) \\
& =\frac{1}{n !}\left(\int_{t}^{T} \lambda(u) d u\right)^{n} \exp \left(-\int_{t}^{T} \lambda(u) d u\right) .
\end{aligned}
$$

According to the reduced-form model, we know that the value of the European vulnerable call option at time 0 is as follows:

$$
C(0, T)=E\left[e^{-r T}\left(S_{T}-K\right)^{+}\left(\omega I_{\{\tau \leq T\}}+I_{\{\tau>T\}}\right) \mid \mathscr{F}_{0}\right] .
$$

where the moment of default is $\tau$, expiration dare is $T$, strike price is $K$, and the asset recovery rate is $\omega$.

Applying the method of structured-form model, let $\omega=$ $((1-w) / D) V_{T}$ and the value of European vulnerable call option at time 0 is given by

$$
\begin{aligned}
& C(0, T)=E\left[e^{-r T}\left(S_{T}-K\right)^{+}\right. \\
& \left.\quad \cdot\left(\frac{1-w}{D} V_{T} I_{\left\{V_{T} \leq D\right\}}+I_{\left\{V_{T}>D\right\}}\right) \mid \mathscr{F}_{0}\right] .
\end{aligned}
$$

We consider to combine the reduced-form model with structured-form model and to define the moment of occurrence of default as the moment of jump or the moment when the default boundary is reached for the first time. We assume that the asset price obeys the mixed fraction Brownian motion and the stock price is subject to the mixed fraction Brownian motion under the jump diffusion model. Then the vulnerable option price model is given by

$$
\begin{aligned}
C & (0, T)=E\left[e^{-r T}\left(S_{T}-K\right)^{+}\right. \\
\cdot & \left.\left(\frac{1-w}{D} V_{T}\left(1-I_{\left\{\tau>T, V_{T}>D\right\}}\right)+I_{\left\{\tau>T, V_{T}>D\right\}}\right) \mid \mathscr{F}_{0}\right] \\
= & E\left[e^{-r T} \frac{1-w}{D} V_{T}\left(S_{T}-K\right)^{+} \mid \mathscr{F}_{0}\right] \\
& +E\left[e^{-r T}\left(S_{T}-K\right)^{+} I_{\left\{\tau>T, V_{T}>D\right\}} \mid \mathscr{F}_{0}\right]-E\left[e^{-r T}\right. \\
& \left.\cdot \frac{1-w}{D} V_{T}\left(S_{T}-K\right)^{+} I_{\left\{\tau>T, V_{T}>D\right\}} \mid \mathscr{F}_{0}\right],
\end{aligned}
$$

where $D$ is constant and denotes the default boundary and $\omega$ follows the assumption that $\omega=((1-w) / D) V_{T}$.

By law of total expectation and Fubini's theorem, we have

$$
\begin{aligned}
E & {\left[e^{-r T}\left(S_{T}-K\right)^{+} I_{\left\{\tau>T, V_{T}>D\right\}} \mid \mathscr{F}_{0}\right] } \\
& =E\left[E\left[e^{-r T}\left(S_{T}-K\right)^{+} I_{\left\{\tau>T, V_{T}>D\right\}} \mid \mathscr{F}_{0}\right] \mid \mathscr{F}_{T}\right] \\
& =E\left[E\left[e^{-r T}\left(S_{T}-K\right)^{+} I_{\left\{\tau>T, V_{T}>D\right\}} \mid \mathscr{F}_{T}\right] \mid \mathscr{F}_{0}\right] \\
& =E\left[e^{-r T}\left(S_{T}-K\right)^{+} E\left[I_{\left\{\tau>T, V_{T}>D\right\}} \mid \mathscr{F}_{T}\right] \mid \mathscr{F}_{0}\right],
\end{aligned}
$$

since the full path of $\{\lambda(t): 0 \leq t \leq T\}$ at time $T$ is known, applying (4), we have [30]

$$
\begin{aligned}
E\left[I_{\{\tau>T\}} \mid \mathscr{F}_{T}\right] & =P(\tau>T)=P\left(N_{T}-N_{0}=0\right) \\
& =\exp \left(-\int_{0}^{T} \lambda(u) d u\right)
\end{aligned}
$$

so

$$
\begin{aligned}
E & {\left[e^{-r T}\left(S_{T}-K\right)^{+} I_{\left\{\tau>T, V_{T}>D\right\}} \mid \mathscr{F}_{0}\right] } \\
& =E\left[e^{-r T-\int_{0}^{T} \lambda(u) d u}\left(S_{T}-K\right)^{+} I_{\left\{V_{T}>D\right\}} \mid \mathscr{F}_{0}\right] .
\end{aligned}
$$

Therefore, (7) can be represented as

$$
\begin{aligned}
& C(0, T)=E\left[e^{-r T} \frac{1-w}{D} V_{T}\left(S_{T}-K\right)^{+} \mid \mathscr{F}_{0}\right] \\
& +E\left[e^{-r T-\int_{0}^{T} \lambda(u) d u}\left(S_{T}-K\right)^{+} I_{\left\{V_{T}>D\right\}} \mid \mathscr{F}_{0}\right] \\
& -E\left[e^{-r T-\int_{0}^{T} \lambda(u) d u} \frac{1-w}{D} V_{T}\left(S_{T}-K\right)^{+} I_{\left\{V_{T}>D\right\}} \mid \mathscr{F}_{0}\right] .
\end{aligned}
$$

We assume that stock price and company's asset price under risk-neutral martingale measure $Q$ obey the next two processes

$$
\begin{aligned}
d S_{t}= & \left(r-\theta \lambda_{1}\right) S_{t} d t+\sigma_{1} S_{t} d W_{1}(t)+\sigma_{1}^{H} S_{t} d W_{1}^{H}(t) \\
& +S_{t} d J(t), \\
d V_{t}= & r V_{t} d t+\sigma_{2} V_{t} d W_{2}(t)+\sigma_{2}^{H} V_{t} d W_{2}^{H}(t),
\end{aligned}
$$

where $J(t)$ is Poisson process and satisfies $J(t)=\sum_{i=1}^{N_{t}} Y_{i}$ and $Y_{i}$ denotes the jump range of J'si - th jump $\left(Y_{0} \stackrel{\sum_{i=1}}{=} 0\right)$. $N_{t}$ denotes Poisson process with a strength of $\lambda_{1}$ and $N_{t}$ is independent of $W_{1}(t), W_{1}^{H}(t) . E\left[Y_{i}\right]=\theta, Y_{1}, Y_{2}, Y_{3}, \cdots$ is a list of independent and identically distributed random variables and is independent of $W_{1}(t), W_{1}^{H}(t), N_{t}$. Assumed that $Y_{i}+1$ 
obeys lognormal distribution, that is, $\ln \left(Y_{i}+1\right) \sim N\left(\mu_{J}, \sigma_{J}^{2}\right)$. By Itố ${ }^{\prime} s$ lemma, we have

$$
\begin{aligned}
S_{T} & =S_{0} \exp \left\{r T-\theta \lambda_{1} T-\frac{1}{2} \sigma_{1}^{2} T+\int_{0}^{T} \sigma_{1} d W_{1}(u)\right. \\
& -\frac{1}{2}\left(\sigma_{1}^{H}\right)^{2} T^{2 H}+\int_{0}^{T} \sigma_{1}^{H} d W_{1}^{H}(u) \\
& \left.+\sum_{i=1}^{N_{T}} \ln \left(Y_{i}+1\right)\right\}, \\
V_{T} & =V_{0} \exp \left\{r T-\frac{1}{2} \sigma_{2}^{2} T+\int_{0}^{T} \sigma_{2} d W_{2}(u)\right. \\
& \left.-\frac{1}{2}\left(\sigma_{2}^{H}\right)^{2} T^{2 H}+\int_{0}^{T} \sigma_{2}^{H} d W_{2}^{H}(u)\right\} .
\end{aligned}
$$

Supposing that default intensity $\lambda(t)$ obeys Vasicek model under risk-neutral measure $Q$

$$
\begin{aligned}
d \lambda(t)= & \alpha[\beta-\lambda(t)] d t+\sigma_{3} d W_{3}(t) \\
& +\sigma_{3}^{H} V_{t} d W_{3}^{H}(t),
\end{aligned}
$$

where $\alpha, \beta, \sigma_{3}$, and $\sigma_{3}^{H}$ are constants and $\lambda(t)$ denotes the intensity of the Cox process characterizing the default process.

The covariance coefficient of $W_{1}(t), W_{2}(t)$, and $W_{3}(t)$ is

$$
\left[\begin{array}{ccc}
1 & \rho_{12} & \rho_{13} \\
\rho_{12} & 1 & \rho_{23} \\
\rho_{13} & \rho_{23} & 1
\end{array}\right] t
$$

and similarly, the covariance coefficient of $W_{1}^{H}(t), W_{2}^{H}(t)$, and $W_{3}^{H}(t)$ is

$$
\left[\begin{array}{ccc}
1 & \rho_{12}^{H} & \rho_{13}^{H} \\
\rho_{12}^{H} & 1 & \rho_{23}^{H} \\
\rho_{13}^{H} & \rho_{23}^{H} & 1
\end{array}\right] t .
$$

\section{Vulnerable Option Pricing Model}

Theorem 1. Let stock price $S_{t}$ and asset price $V_{t}$ satisfy (12) and (13) and the default intensity $\lambda(t)$ satisfies (16); then the value of the European vulnerable call option at time 0 is

$$
\begin{aligned}
C & (0, T)=\sum_{n=0}^{\infty} \frac{\left(\lambda_{2} T\right)^{n}}{n !} e^{-\lambda_{2} T}\left[\frac{1-w}{D} S_{0} V_{0}\right. \\
\cdot & \exp \left(r T+\rho_{12} \sigma_{1} \sigma_{2} T+\rho_{12}^{H} \sigma_{1}^{H} \sigma_{2}^{H} T^{2 H}\right) N\left(d_{1}(n)\right) \\
+ & e^{-r T} A(0, T) N\left(d_{3}(n), d_{4}(n),\left(\rho_{12}, \rho_{12}^{H}\right)\right)-e^{-r T} \\
& \cdot \frac{1-w}{D} K A(0, T) Z(0, T) \\
& \left.\cdot N\left(d_{7}(n), d_{8}(n),\left(\rho_{12}, \rho_{12}^{H}\right)\right)\right]-\sum_{n=0}^{\infty} \frac{\left(\lambda_{1} T\right)^{n}}{n !} e^{-\lambda_{1} T} \\
& \cdot\left[\frac{1-w}{D} K V_{0} N\left(d_{2}(n)\right)+e^{-r T} K A(0, T)\right. \\
& \cdot N\left(d_{5}(n), d_{6}(n),\left(\rho_{12}, \rho_{12}^{H}\right)\right)-e^{r T} \frac{1-w}{D} \\
& \left.\cdot K A(0, T) Y(0, T) N\left(d_{9}(n), d_{1} 0(n),\left(\rho_{12}, \rho_{12}^{H}\right)\right)\right],
\end{aligned}
$$

where $K$ is strike price, $r$ is the riskless interest rate, $T$ is maturity, $D$ is default boundary, $((1-w) / D) V_{T}$ is default recovery rate, and $\sigma_{1}, \sigma_{2}, \sigma_{1}^{H}$, and $\sigma_{2}^{H}$ are constant,

$$
\begin{aligned}
& A(0, T)=\exp \left\{-\beta T-[\lambda(0)-b] M(0, T, \alpha)+\frac{1}{2}{\sigma_{3}}^{2} \int_{0}^{T} M^{2}(u, T, \alpha) d u+H\left(\sigma_{3}^{H}\right)^{2} \int_{0}^{T} M(u, T, \alpha) u^{2 H-1} d u\right\}, \\
& X(0, T)=S_{0} \exp \left\{r T-\rho_{13} \sigma_{1} \sigma_{3} \int_{0}^{T} M(u, T, \alpha) d u-2 H \rho_{13}^{H} \sigma_{1}^{H} \sigma_{3}^{H} \int_{0}^{T} M(u, T, \alpha) u^{2 H-1} d u\right\}, \\
& Y(0, T)=V_{0} \exp \left\{r T-\rho_{23} \sigma_{2} \sigma_{3} \int_{0}^{T} M(u, T, \alpha) d u-2 H \rho_{23}^{H} \sigma_{2}^{H} \sigma_{3}^{H} \int_{0}^{T} M(u, T, \alpha) u^{2 H-1} d u\right\}, \\
& Z(0, T)=S_{0} V_{0} \exp \left\{2 r T-\left(\rho_{12} \sigma_{1}+\rho_{23} \sigma_{2}\right) \sigma_{3} \int_{0}^{T} M(u, T, \alpha) d u+\rho_{12} \sigma_{1} \sigma_{2}+\rho_{12}^{H} \sigma_{1}^{H} \sigma_{2}^{H}\right. \\
& \left.-2 H\left(\rho_{12}^{H} \sigma_{1}^{H}+\rho_{23}^{H} \sigma_{2}^{H}\right) \sigma_{3}^{H} \int_{0}^{T} M(u, T, \alpha) u^{2 H-1} d u\right\}, \\
& d_{1}(n)=\frac{\ln \left(S_{0} / K\right)+r T+(1 / 2) \sigma_{1}^{2} T+(1 / 2)\left(\sigma_{1}^{H}\right)^{2} T^{2 H}+\rho_{12} \sigma_{1} \sigma_{2} T+\rho_{12}^{H} \sigma_{1}^{H} \sigma_{2}^{H}-\theta \lambda_{2} T+n \mu_{J}}{\sqrt{\sigma_{1}^{2} T+\left(\sigma_{1}^{H}\right)^{2} T^{2 H}+n \sigma_{J}{ }^{2}}}, \\
& d_{2}(n)=d_{1}(n)-\sqrt{\sigma_{1}^{2} T+\left(\sigma_{1}^{H}\right)^{2} T^{2 H}+n \sigma_{J}^{2}}+\frac{n \sigma_{I}^{2}+\left(\lambda_{2}-\lambda_{1}\right) \theta T}{\sqrt{\sigma_{1}^{2} T+\left(\sigma_{1}^{H}\right)^{2} T^{2 H}+n \sigma_{J}^{2}}},
\end{aligned}
$$




$$
\begin{aligned}
& d_{3}(n)=\frac{\ln \left(S_{0} / K\right)+r T+(1 / 2) \sigma_{1}^{2} T-\rho_{13} \sigma_{1} \sigma_{3} \int_{0}^{T} M(u, T, \alpha) d u+(1 / 2)\left(\sigma_{1}^{H}\right)^{2} T^{2 H}}{\sqrt{\sigma_{1}^{2} T+\left(\sigma_{1}^{H}\right)^{2} T^{2 H}+n \sigma_{J}^{2}}} \\
& +\frac{-2 H \rho_{13}^{H} \sigma_{1}^{H} \sigma_{3}^{H} \int_{0}^{T} M(u, T, \alpha) u^{2 H-1} d u-\theta \lambda_{2} T+n \mu_{J}}{\sqrt{\sigma_{1}^{2} T+\left(\sigma_{1}^{H}\right)^{2} T^{2 H}+n \sigma_{J}^{2}}}, \\
& d_{4}(n)=\frac{\ln \left(V_{0} / D\right)+r T-(1 / 2) \sigma_{2}{ }^{2} T+\rho_{12} \sigma_{1} \sigma_{2} T-\rho_{23} \sigma_{2} \sigma_{3} \int_{0}^{T} M(u, T, \alpha) d u-(1 / 2)\left(\sigma_{2}^{H}\right)^{2} T^{2 H}}{\sqrt{\sigma_{2}{ }^{2} T+\left(\sigma_{2}^{H}\right)^{2} T^{2 H}}} \\
& +\frac{\rho_{12}^{H} \sigma_{1}^{H} \sigma_{2}^{H} T^{2 H}-2 H \rho_{23}^{H} \sigma_{2}^{H} \sigma_{3}^{H} \int_{0}^{T} M(u, T, \alpha) u^{2 H-1} d u}{\sqrt{\sigma_{2}^{2} T+\left(\sigma_{2}^{H}\right)^{2} T^{2 H}}}, \\
& d_{5}(n)=d_{3}(n)-\frac{\sigma_{1}^{2} T+(1 / 2)\left(\sigma_{1}^{H}\right)^{2}\left(T+T^{2 H}\right)+\left(\lambda_{1}-\lambda_{2}\right) \theta T}{\sqrt{\sigma_{1}^{2} T+\left(\sigma_{1}^{H}\right)^{2} T^{2 H}+n \sigma_{J}^{2}}}, \\
& d_{6}(n)=d_{4}(n)-\frac{\rho_{12} \sigma_{1} \sigma_{2} T+\rho_{12}^{H} \sigma_{1}^{H} \sigma_{2}^{H} T^{2 H}}{\sqrt{\sigma_{2}^{2} T+\left(\sigma_{2}^{H}\right)^{2} T^{2 H}}}, \\
& d_{7}(n)=d_{3}(n)+\frac{\rho_{12} \sigma_{1} \sigma_{2} T+\rho_{12}^{H} \sigma_{1}^{H} \sigma_{2}^{H} T^{2 H}}{\sqrt{\sigma_{1}{ }^{2} T+\left(\sigma_{1}^{H}\right)^{2} T^{2 H}+n \sigma_{J}^{2}}}, \\
& d_{8}(n)=d_{4}(n)+\sqrt{\sigma_{2}{ }^{2} T+\left(\sigma_{2}^{H}\right)^{2} T^{2 H}}, \\
& d_{9}(n)=d_{3}(n)-\frac{\sigma_{1}^{2} T+\left(\sigma_{1}^{H}\right)^{2} T^{2 H}-\rho_{12} \sigma_{1} \sigma_{2} T-\left(\lambda_{1}-\lambda_{2}\right) \theta T}{\sqrt{\sigma_{1}^{2} T+\left(\sigma_{1}^{H}\right)^{2} T^{2 H}+n \sigma_{J}^{2}}}, \\
& d_{10}(n)=d_{6}(n)+\sqrt{\sigma_{2}^{2} T+\left(\sigma_{2}^{H}\right)^{2} T^{2 H}}, \\
& \lambda_{2}=(1+\theta) \lambda_{1} \text {, } \\
& N(x, y, \rho)=\int_{-\infty}^{x} \int_{-\infty}^{y} \frac{1}{2 \pi \sigma_{x} \sigma_{y} \sqrt{1-\rho^{2}}} \exp \left\{-\frac{1}{2(1-\rho)}\left[\frac{\left(x-\mu_{x}\right)^{2}}{\sigma_{x}{ }^{2}}+\frac{\left(y-\mu_{y}\right)^{2}}{\sigma_{y}{ }^{2}}-\frac{2 \rho\left(x-\mu_{x}\right)\left(y-\mu_{y}\right)}{\sigma_{x} \sigma_{y}}\right]\right\} d x d y,
\end{aligned}
$$

$(X, Y)$ obey two-dimensional normal distribution, i.e., $(X, Y) \sim N\left(\mu_{x}, \sigma_{x}{ }^{2} ; \mu_{y}, \sigma_{y}{ }^{2} ; \rho\right)$, where $\mu_{x}, \mu_{y}, \sigma_{x}, \sigma_{y}$, and $\rho$ are constant and $\sigma_{x}>0, \sigma_{y}>0$, and $-1<\rho<1$.

Proof. For convenience, (11) can be written as

$$
\begin{aligned}
& C(0, T)=E\left[e^{-r T} \frac{1-w}{D} V_{T}\left(S_{T}-K\right)^{+} \mid \mathscr{F}_{0}\right] \\
& +E\left[e^{-r T-\int_{0}^{T} \lambda(u) d u}\left(S_{T}-K\right)^{+} I_{\left\{V_{T}>D\right\}} \mid \mathscr{F}_{0}\right] \\
& \quad-E\left[e^{-r T-\int_{0}^{T} \lambda(u) d u} \frac{1-w}{D} V_{T}\left(S_{T}-K\right)^{+} I_{\left\{V_{T}>D\right\}} \mid \mathscr{F}_{0}\right] \\
& \quad=I+I I-I I I,
\end{aligned}
$$

where

$$
\begin{aligned}
& I=E\left[e^{-r T} \frac{1-w}{D} V_{T}\left(S_{T}-K\right)^{+} \mid \mathscr{F}_{0}\right], \\
& I I=E\left[e^{-r T-\int_{0}^{T} \lambda(u) d u}\left(S_{T}-K\right)^{+} I_{\left\{V_{T}>D\right\}} \mid \mathscr{F}_{0}\right], \\
& I I I=E\left[e^{-r T-\int_{0}^{T} \lambda(u) d u} \frac{1-w}{D} V_{T}\left(S_{T}-K\right)^{+}\right. \\
& \left.\cdot I_{\left\{V_{T}>D\right\}} \mid \mathscr{F}_{0}\right] .
\end{aligned}
$$

By calculating, we have

$$
I=\frac{1-w}{D} S_{0} V_{0} \exp \left(r T+\rho_{12} \sigma_{1} \sigma_{2} T+\rho_{12}^{H} \sigma_{1}^{H} \sigma_{2}^{H} T^{2 H}\right)
$$




$$
\begin{aligned}
& \cdot \sum_{n=0}^{\infty} \frac{\left(\lambda_{2} T\right)^{n}}{n !} e^{-\lambda_{2} T} N\left(d_{1}(n)\right)-\frac{1-w}{D} K V_{0} \\
& \cdot \sum_{n=0}^{\infty} \frac{\left(\lambda_{1} T\right)^{n}}{n !} e^{-\lambda_{1} T} N\left(d_{2}(n)\right), \\
I I= & e^{-r T} A(0, T) X(0, T) \\
& \cdot \sum_{n=0}^{\infty} \frac{\left(\lambda_{2} T\right)^{n}}{n !} e^{-\lambda_{2} T} N\left(d_{3}(n), d_{4}(n),\left(\rho_{12}, \rho_{12}^{H}\right)\right) \\
& -e^{-r T} K A(0, T) \\
& \cdot \sum_{n=0}^{\infty} \frac{\left(\lambda_{1} T\right)^{n}}{n !} e^{-\lambda_{1} T} N\left(d_{5}(n), d_{6}(n),\left(\rho_{12}, \rho_{12}^{H}\right)\right), \\
I I I & =e^{-r T} \frac{1-w}{D} K A(0, T) Z(0, T) \\
& \cdot \sum_{n=0}^{\infty} \frac{\left(\lambda_{1} T\right)^{n}}{n !} e^{-\lambda_{1} T} N\left(d_{9}(n), d_{10}(n),\left(\rho_{12}, \rho_{12}^{H}\right)\right) . \\
& \sum_{n=0} \frac{\left(\lambda_{2} T\right)^{n}}{n !} e^{-\lambda_{2} T} N\left(d_{7}(n), d_{8}(n),\left(\rho_{12}, \rho_{12}^{H}\right)\right) \\
& =w \\
& =1-T) Y(0, T)
\end{aligned}
$$

We show the derivation process of $I$. For $I I$ and $I I I$, the derivation process is included in the Appendix A and B which are similar to $I$. We define

$$
I=E\left[e^{-r T} \frac{1-w}{D} V_{T}\left(S_{T}-K\right)^{+} \mid \mathscr{F}_{0}\right]=I_{1}-I_{2},
$$

where

$$
\begin{aligned}
& I_{1}=E\left[e^{-r T} \frac{1-w}{D} S_{T} V_{T} 1_{\left\{S_{T}>K\right\}} \mid \mathscr{F}_{0}\right], \\
& I_{2}=E\left[e^{-r T} \frac{1-w}{D} K V_{T} 1_{\left\{S_{T}>K\right\}} \mid \mathscr{F}_{0}\right] .
\end{aligned}
$$

Step one, we calculate $I_{2}$. At first, we introduce a new measure

$$
\begin{aligned}
& \frac{d Q^{V}}{d Q} \mid \mathscr{F}_{0}=\frac{V_{T}}{E\left[V_{T} \mid \mathscr{F}_{0}\right]}=\exp \left\{\int_{0}^{T} \sigma_{2} d W_{2}(u)\right. \\
& \left.-\frac{1}{2} \int_{0}^{T} \sigma_{2}^{2} d u+\int_{0}^{T} \sigma_{2}^{H} d W_{2}^{H}(u)-\frac{1}{2}\left(\sigma_{2}^{H}\right)^{2} T^{2 H}\right\} .
\end{aligned}
$$

Substituting (15) into $I_{2}$, we have

$$
\begin{aligned}
I_{2} & =\frac{1-w}{D} K V_{0} E\left[\operatorname { e x p } \left\{\int_{0}^{T} \sigma_{2} d W_{2}(u)-\frac{1}{2} \int_{0}^{T} \sigma_{2}^{2} d u\right.\right. \\
& \left.\left.+\int_{0}^{T} \sigma_{2}^{H} d W_{2}^{H}(u)-\frac{1}{2}\left(\sigma_{2}^{H}\right)^{2} T^{2 H}\right\} \cdot I_{\left\{S_{T}>K\right\}} \mid \mathscr{F}_{0}\right] \\
& =\frac{1-w}{D} K V_{0} E^{V}\left[I_{\left\{S_{T}>K\right\}} \mid \mathscr{F}_{0}\right] .
\end{aligned}
$$

For $\left(d Q^{V} / d Q\right) \mid \mathscr{F}_{0}$ satisfying Girsanov theorem, we have

$$
\begin{gathered}
W_{1}^{V}(T)=W_{1}(T)-\rho_{12} \sigma_{2} T, \\
W_{2}^{V}(T)=W_{2}(T)-\sigma_{2} T, \\
W_{1}^{H V}(T)=W_{1}^{H}(T)-\rho_{12}^{H} \sigma_{2}^{H} T^{2 H}, \\
W_{2}^{H V}(T)=W_{2}^{H}(T)-\sigma_{2}^{H} T^{2 H},
\end{gathered}
$$

thus under $Q^{V}$ measure, (14) can be written as

$$
\begin{aligned}
S_{T} & =S_{0} \exp \left\{r T-\frac{1}{2} \sigma_{1}^{2} T+\int_{0}^{T} \sigma_{1} d W_{1}(u)\right. \\
& -\frac{1}{2}\left(\sigma_{1}^{H}\right)^{2} T^{2 H}+\int_{0}^{T} \sigma_{1}^{H} d W_{1}^{H}(u)-\theta \lambda_{1} T \\
& \left.+\sum_{i=0}^{N_{T}} \ln \left(Y_{i}+1\right)\right\}=S_{0} \exp \left\{r T-\frac{1}{2} \sigma_{1}^{2} T\right. \\
& +\int_{0}^{T} \sigma_{1} d W_{1}^{Y}(u)+\rho_{12} \sigma_{1} \sigma_{2} T-\frac{1}{2}\left(\sigma_{1}^{H}\right)^{2} T^{2 H} \\
& +\int_{0}^{T} \sigma_{1}^{H} d W_{1}^{H Y}(u)+\rho_{12}^{H} \sigma_{1}^{H} \sigma_{2}^{H} T^{2 H}-\theta \lambda_{1} T \\
& \left.+\sum_{i=0}^{N_{T}} \ln \left(Y_{i}+1\right)\right\},
\end{aligned}
$$

for

$$
\begin{gathered}
\int_{0}^{T} \sigma_{1} d W_{1}^{V}(u) \sim N\left(0, \sigma_{1}^{2} T\right), \\
\int_{0}^{T} \sigma_{1}^{H} d W_{1}^{H V}(u) \sim N\left(0,\left(\sigma_{1}^{H}\right)^{2} T^{2 H}\right),
\end{gathered}
$$

and when $N_{T}=n$, we have

$$
\sum_{i=0}^{N_{t}} \ln \left(Y_{i}+1\right) \sim N\left(n \mu_{J}, n \sigma_{J}^{2}\right)
$$

thus

$$
E^{V}\left[I_{\left\{S_{T}>K\right\}} \mid \mathscr{F}_{0}\right]=\sum_{n=0}^{\infty} \frac{\left(\lambda_{1} T\right)^{n}}{n !} e^{-\lambda_{1} T} N\left(d_{2}(n)\right),
$$

then (30) is written as

$$
I_{2}=\frac{1-w}{D} K V_{0} \sum_{n=0}^{\infty} \frac{\left(\lambda_{1} T\right)^{n}}{n !} e^{-\lambda_{1} T} N\left(d_{2}(n)\right)
$$

where 


$$
d_{2}(n)=\frac{\ln \left(S_{0} / K\right)+r T-(1 / 2) \sigma_{1}^{2} T-(1 / 2)\left(\sigma_{1}^{H}\right)^{2} T^{2 H}+\rho_{12} \sigma_{1} \sigma_{2} T+\rho_{12}^{H} \sigma_{1}^{H} \sigma_{2}^{H} T^{2 H}-\theta \lambda_{1} T+n \mu_{J}}{\sqrt{\sigma_{1}^{2} T+\left(\sigma_{1}^{H}\right)^{2} T^{2 H}+n \sigma_{J}^{2}}} .
$$

Step two, we calculate $I_{1}$. At first we introduce a new measure

$$
\begin{aligned}
& \frac{d Q^{S V}}{d Q} \mid \mathscr{F}_{0}=\frac{S_{T} V_{T}}{E\left[S_{T} V_{T} \mid \mathscr{F}_{0}\right]}=\exp \left\{\int_{0}^{T} \sigma_{1} d W_{1}(u)\right. \\
& +\int_{0}^{T} \sigma_{2} d W_{2}(u)-\frac{1}{2}\left(\sigma_{1}^{2}+\sigma_{2}^{2}+2 \rho_{12} \sigma_{1} \sigma_{2}\right) T \\
& +\int_{0}^{T} \sigma_{2}^{H} d W_{2}^{H}(u) \\
& -\frac{1}{2}\left(\left(\sigma_{1}^{H}\right)^{2}+\left(\sigma_{2}^{H}\right)^{2}+2 \rho_{12}^{H} \sigma_{1}^{H} \sigma_{2}^{H}\right) T^{2 H} \\
& \left.+\int_{0}^{T} \sigma_{1}^{H} d W_{1}^{H}(u)-\theta \lambda_{1} T+\sum_{i=0}^{N_{T}} \ln \left(Y_{i}+1\right)\right\}
\end{aligned}
$$

By multidimensional Girsanov theorem, we get

$$
\begin{gathered}
W_{1}^{S V}(T)=W_{1}(T)-\sigma_{1} T-\rho_{12} \sigma_{2} T, \\
\left(W_{1}^{H}\right)^{S V}(T)=W_{1}^{H}(T)-\sigma_{1}^{H} T^{2 H}-\rho_{12}^{H} \sigma_{2}^{H} T^{2 H},
\end{gathered}
$$

under $Q^{S V}$ measure, (14) can be written as

$$
\begin{aligned}
S_{T} & =S_{0} \exp \left\{r T+\frac{1}{2} \sigma_{1}^{2} T+\int_{0}^{T} \sigma_{1} d W_{1}^{S V}(u)\right. \\
& +\rho_{12} \sigma_{1} \sigma_{2} T-\frac{1}{2}\left(\sigma_{1}^{H}\right)^{2} T^{2 H}
\end{aligned}
$$

$$
\begin{aligned}
& +\int_{0}^{T}\left(\sigma_{1}^{H}\right)^{2} d\left(W_{1}^{H}\right)^{S V}(u)+\rho_{12}^{H} \sigma_{1}^{H} \sigma_{2}^{H} T^{2 H}-\theta \lambda_{2} T \\
& \left.+\sum_{i=0}^{N_{T}} \ln \left(Y_{i}+1\right)\right\} .
\end{aligned}
$$

By introducing Radon - Nikodym derivative, we have

$$
\begin{aligned}
I_{1} & =E\left[e^{-r T} \frac{1-w}{D} S_{T} V_{T} I_{\left\{S_{T}>K\right\}} \mid \mathscr{F}_{0}\right]=e^{-r T} \frac{1-w}{D} \\
& \cdot E\left[S_{T} V_{T} I_{\left\{S_{T}>K\right\}} \mid \mathscr{F}_{0}\right]=\frac{1-w}{D} S_{0} V_{0} \\
& \cdot \exp \left(r T+\rho_{12} \sigma_{1} \sigma_{2} T+\rho_{12}^{H} \sigma_{1}^{H} \sigma_{2}^{H} T^{2 H}\right) \\
& \cdot E^{S V}\left[I_{\left\{S_{T}>K\right\}} \mid \mathscr{F}_{0}\right],
\end{aligned}
$$

and similarly, when $N_{T}=n$, there exists

$$
E^{S V}\left[I_{\left\{S_{T}>K\right\}} \mid \mathscr{F}_{0}\right]=\sum_{n=0}^{\infty} \frac{\left(\lambda_{2} T\right)^{n}}{n !} e^{-\lambda_{2} T} N\left(d_{1}(n)\right),
$$

and hence we have

$$
\begin{aligned}
I_{1}= & \frac{1-w}{D} S_{0} V_{0} \exp \left(r T+\rho_{12} \sigma_{1} \sigma_{2} T+\rho_{12}^{H} \sigma_{1}^{H} \sigma_{2}^{H} T^{2 H}\right) \\
& \cdot \sum_{n=0}^{\infty} \frac{\left(\lambda_{2} T\right)^{n}}{n !} e^{-\lambda_{2} T} N\left(d_{1}(n)\right),
\end{aligned}
$$

where

$$
d_{1}(n)=\frac{\ln \left(S_{0} / K\right)+r T+(1 / 2) \sigma_{1}^{2} T+(1 / 2)\left(\sigma_{1}^{H}\right)^{2} T^{2 H}+\rho_{12} \sigma_{1} \sigma_{2} T+\rho_{12}^{H} \sigma_{1}^{H} \sigma_{2}^{H} T^{2 H}-\theta \lambda_{2} T+n \mu_{J}}{\sqrt{\sigma_{1}^{2} T+\left(\sigma_{1}^{H}\right)^{2} T^{2 H}+n \sigma_{J}^{2}}} .
$$

Therefore, we have

$$
I=I_{1}+I_{2} .
$$

The proof is finished.

\section{Numerical Test}

The images of option value under different parameters with respect to the underlying asset price changes are given respectively in order to investigate the influence of various parameters on the value of the vulnerable option. Assuming that each parameter has the following values: the constant interest rate is $r=0.05$, the strike price is $K=100$, the maturity is $T=1$, the default threshold is $D=30$, the expected value of jump range is $\theta=0.25$, the percentage of the cost of the bankruptcy to the option seller's asset is $w=0.3$, the stock price volatility under standard Brownian motion are $\sigma_{1}=0.5, \sigma_{2}=0.3, \sigma_{3}=0.3$, the stock price volatility under fractional Brownian motion are $\sigma_{1}^{H}=0.5$, $\sigma_{2}^{H}=0.3, \sigma_{3}^{H}=0.3$, the expectation and standard deviation of $\ln \left(Y_{i}+1\right)$ are $\mu_{J}=0.2, \sigma_{J}=0.1$, the correlation coefficients between $W_{1}(t), W_{2}(t), W_{3}(t)$ and $W_{1}^{H}(t), W_{2}^{H}(t), W_{3}^{H}(t)$ are $\rho_{12}=\rho_{13}=\rho_{23}=\rho_{12}^{H}=\rho_{13}^{H}=\rho_{23}^{H}=0.5$. 


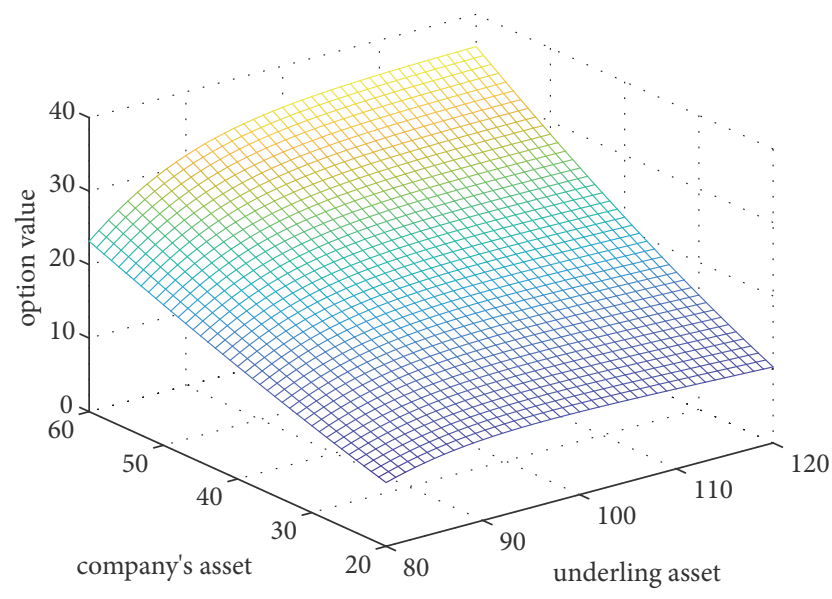

FIGURE 1: Change of option value.

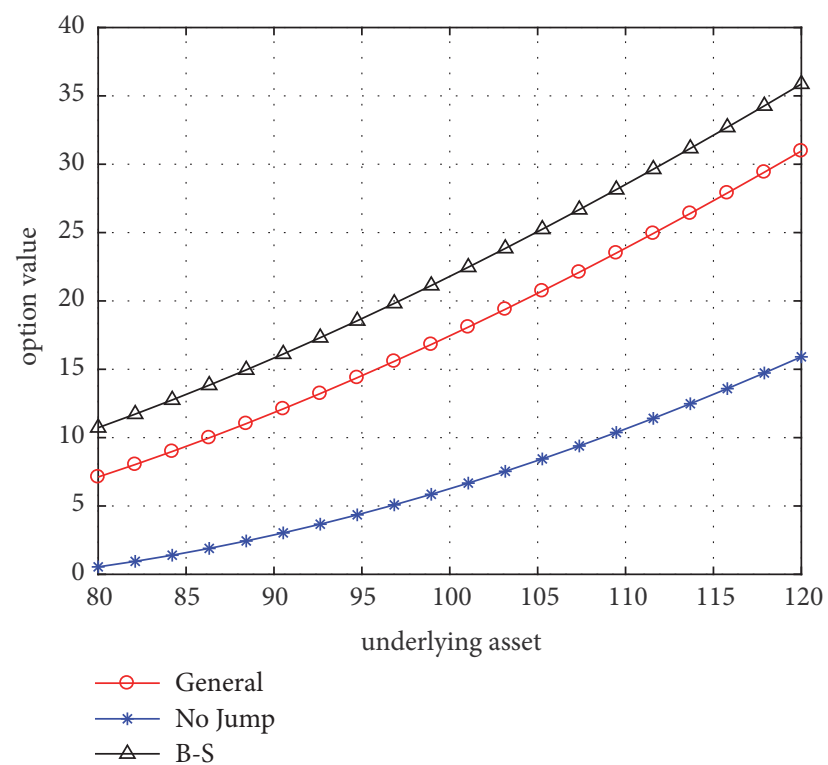

FIGURE 2: Change of option value under different pricing methods.

The value of vulnerable option is obtained from (19). By using Matlab software, we show some results of numerical tests.

Figure 1 shows that the value of the option changes with both underlying asset and the price of the company's asset. As we can see from the figure, the option value increases with the increase of the underlying asset price and the company asset price. The underlying asset price is positively correlated with the company asset price and the option value.

From Figure 2, when there is a jump process that the jump range expectation is positive, the option value is higher than the normal one and this is in line with the actual situation. This situation shows that the jump process has a significant influence on option pricing. In actual market, introducing jump process can make option pricing more accurate. Since in this article the situation of default is considered, the value of the option is slightly lower than the value of the classical Black-Scholes option and this is also consistent with the

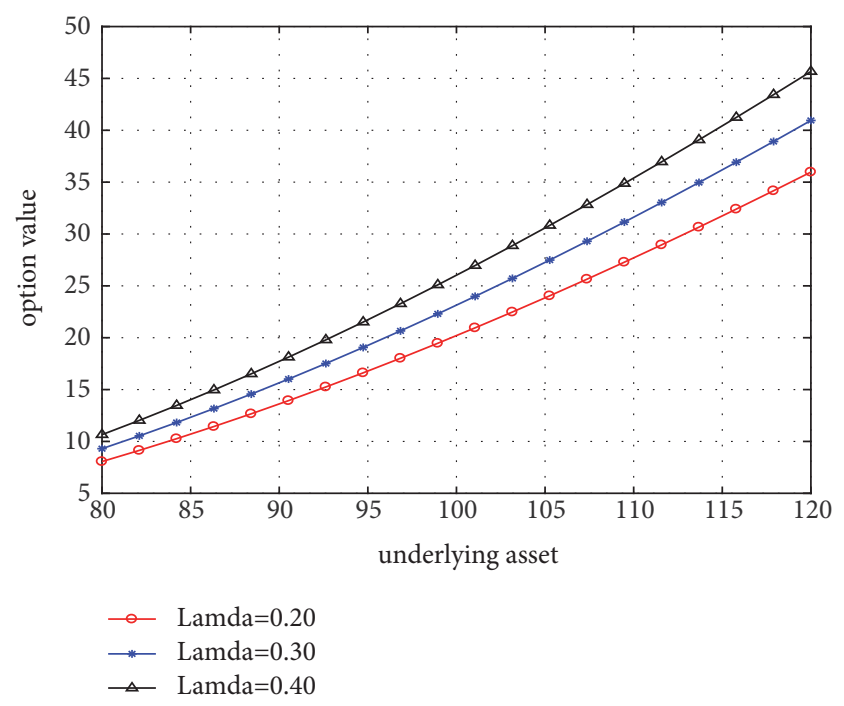

FIGURE 3: Different default intensity.

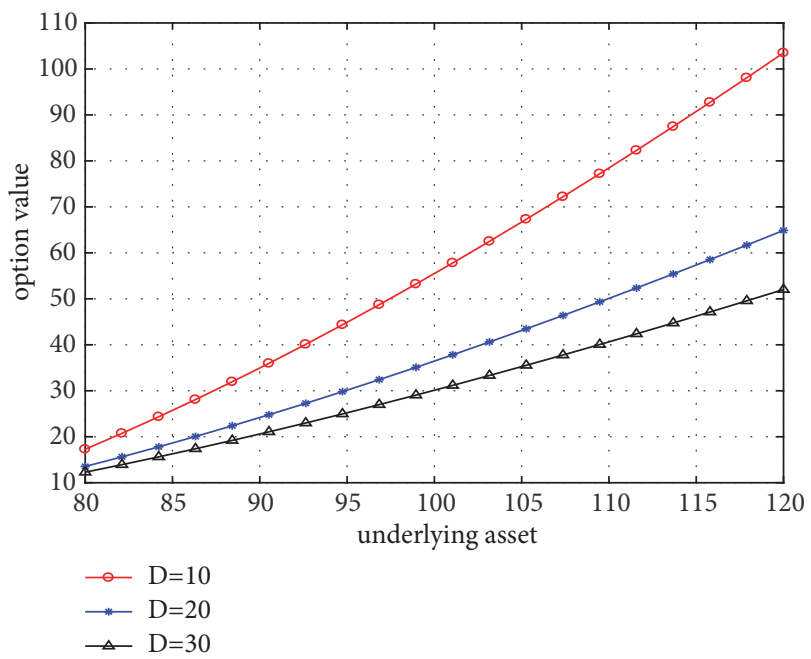

FIGURE 4: Different boundaries.

actual situation. The introduction of default in this article can make option pricing more perfect.

Figures 3 and 4 depict the relationship between option value and underlying asset under different parameters respectively. Figure 3 depicts the value of options under different default intensity and the figure shows that as the default intensity increases, the option value is also growing. Figure 4 depicts the change of option value under different boundaries. From the figure, as the default boundary increases, the option value gradually decreases due to the increased default risk. This situation agrees with the actual market.

Figure 5 depicts the effect of the correlation coefficients $\rho_{12}$ and $\rho_{12}^{H}$ on the price of the option. As we can see from Figure 5, as the correlation coefficient increases, the option value continues to decrease. However, the correlation coefficient in (a) has a greater impact on option price than that in (b). 


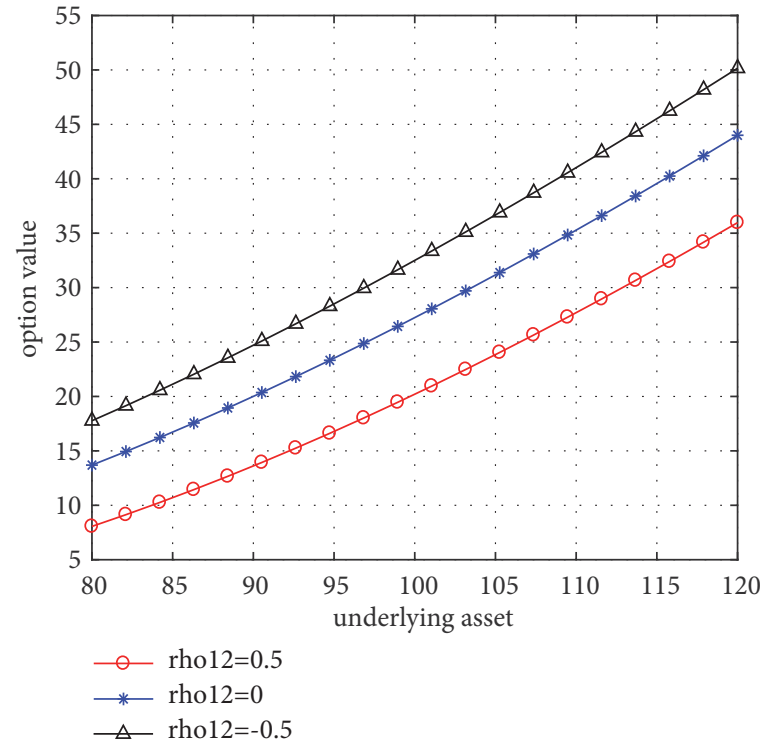

(a) $\rho_{12}$

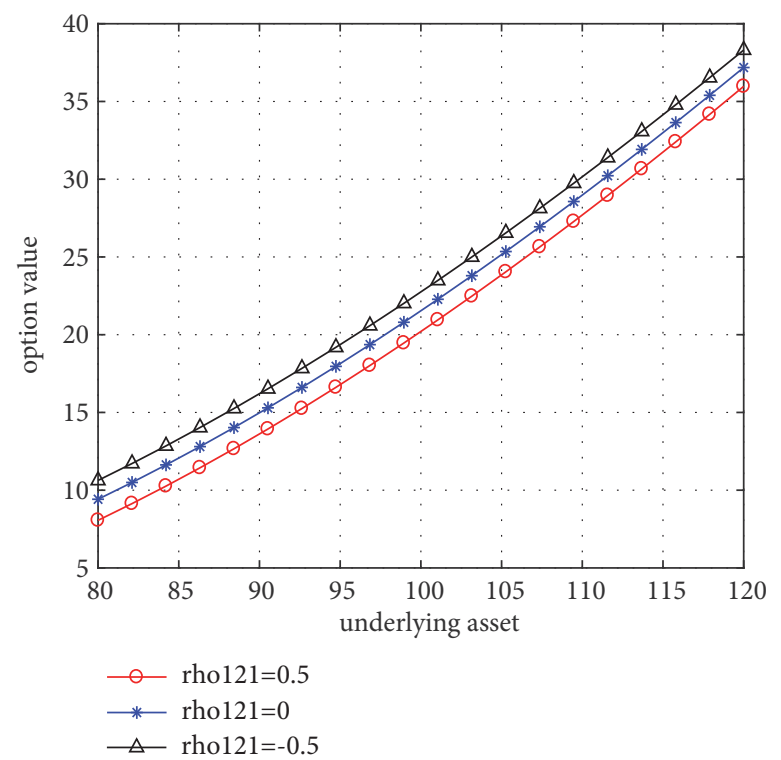

(b) $\rho_{12}^{H}$

Figure 5: Change of option value under different correlation coefficient.

\section{Conclusion}

The traditional definition of default probability is divided into two types: reduced-form model and structured-form model. The reduced-form model supposes that default occurs only when jump occurs and structured-form model supposes that default occurs when the company's asset value is less than the default boundary. The probability of default is determined by the relationship between the company's asset value and default boundary. We employ the combination of reducedform model and structured-form model to pricing European vulnerable option. In this paper, it is assumed that the default can be caused by jump process or by the insolvent of company. The expression of vulnerable option value is given under the condition of risk-free interest rate and the analytical solution is obtained. Finally, we conduct numerical experiments on the analytical solutions and analyze the results.

\section{Appendix}

\section{A. Derivation Process of II}

Derived of $I I$. Define

$$
\begin{aligned}
I I & =E\left[e^{-r T-\int_{0}^{T} \lambda(u) d u}\left(S_{T}-K\right)^{+} I_{\left\{V_{T}>D\right\}} \mid \mathscr{F}_{0}\right] \\
& =I_{3}-I_{4},
\end{aligned}
$$

where

$$
\begin{aligned}
& I_{3}=E\left[e^{-r T-\int_{0}^{T} \lambda(u) d u} S_{T} I_{\left\{S_{T}>K, V_{T}>D\right\}} \mid \mathscr{F}_{0}\right], \\
& I_{4}=E\left[e^{-r T-\int_{0}^{T} \lambda(u) d u} K I_{\left\{S_{T}>K, V_{T}>D\right\}} \mid \mathscr{F}_{0}\right] .
\end{aligned}
$$

Step one, we calculus $I_{4}$. The equivalent martingale measure of $Q^{\lambda}$ defined by derivative definition $Q$ of RadonNikodym as follows

$$
\frac{d Q^{\lambda}}{d Q} \mid \mathscr{F}_{0}=\frac{e^{-\int_{0}^{T} \lambda(u) d u}}{E\left[e^{-\int_{0}^{T} \lambda(u) d u} \mid \mathscr{F}_{0}\right]} .
$$

Solving the solution of Equation (16), we derive

$$
\begin{aligned}
\lambda(t)= & e^{-\alpha(t-s)} \lambda(s)+\beta\left(1-e^{-\alpha(t-s)}\right) \\
& +\sigma_{3} \int_{s}^{t} e^{-\alpha(t-u)} d W_{3}(u) \\
& +\sigma_{3}^{H} \int_{s}^{t} e^{-\alpha(t-u)} d W_{3}^{H}(u),
\end{aligned}
$$

we integrate two sides from $[t, T]$ exclusively

$$
\begin{aligned}
\int_{t}^{T} \lambda(s) d s= & \beta(T-t)+[\lambda(t)-\beta] \int_{t}^{T} e^{-\alpha(s-t)} d s \\
& +\int_{t}^{T} \sigma_{3} \int_{t}^{s} e^{-\alpha(s-u)} d W_{3}(u) d s \\
& +\int_{t}^{T} \sigma_{3}^{H} \int_{t}^{s} e^{-\alpha(s-u)} d W_{3}^{H}(u) d s,
\end{aligned}
$$

applying Fubini's theorem, we obtain

$$
\begin{aligned}
\int_{t}^{T} \lambda(s) d s= & \beta(T-t)+\frac{1}{\alpha}[\lambda(t)-\beta]\left(1-e^{-\alpha(T-t)}\right) \\
& +\sigma_{3} \int_{t}^{T} \int_{t}^{s} e^{-\alpha(s-u)} d W_{3}(u) d s \\
& +\sigma_{3}^{H} \int_{t}^{T} \int_{t}^{s} e^{-\alpha(s-u)} d W_{3}^{H}(u) d s
\end{aligned}
$$




$$
\begin{aligned}
= & \beta(T-t)+\frac{1}{\alpha}[\lambda(t)-\beta]\left(1-e^{-\alpha(T-t)}\right) \\
& +\frac{1}{\alpha} \sigma_{3} \int_{t}^{s}\left(1-e^{-\alpha(T-u)}\right) d W_{3}(u) \\
& +\frac{1}{\alpha} \sigma_{3}^{H} \int_{t}^{s}\left(1-e^{-\alpha(T-u)}\right) d W_{3}^{H}(u),
\end{aligned}
$$

$$
\begin{aligned}
& -\sigma_{3}^{H} \int_{0}^{T} M(u, T, \alpha) d W_{3}^{H}(u) \\
& -\frac{1}{2} \sigma_{3}^{2} \int_{0}^{T} M^{2}(u, T, \alpha) d u \\
& \left.-H\left(\sigma_{3}^{H}\right)^{2} \int_{0}^{T} M(u, T, \alpha) u^{2 H-1} d u\right\} .
\end{aligned}
$$

let

$$
\begin{aligned}
& M(0, T, \alpha)=\frac{1}{\alpha}\left(1-e^{-\alpha T}\right), \\
& M(0, T, a)=\frac{1}{a}\left(1-e^{-a T}\right),
\end{aligned}
$$

where $t=0$ is given as

$$
\begin{aligned}
\int_{0}^{T} \lambda(s) d s= & \beta T+[\lambda(0)-\beta] M(0, T, \alpha) \\
& +\sigma_{3} \int_{0}^{s} M(u, T, \alpha) d W_{3}(u) \\
& +\sigma_{3}^{H} \int_{0}^{s} M(u, T, \alpha) d W_{3}^{H}(u), \\
-\int_{0}^{T} \lambda(s) d s= & -\beta T-[\lambda(0)-\beta] M(0, T, \alpha) \\
& -\sigma_{3} \int_{0}^{s} M(u, T, \alpha) d W_{3}(u) \\
& -\sigma_{3}^{H} \int_{0}^{s} M(u, T, \alpha) d W_{3}^{H}(u),
\end{aligned}
$$

let

$$
A(0, T)=E\left[e^{-\int_{0}^{T} \lambda(u) d u} \mid \mathscr{F}_{0}\right]
$$

using multidimensional fractional $I t \widehat{o}^{\prime} s$ lemma

$$
\begin{aligned}
& A(0, T)=\exp \{-\beta T-[\lambda(0)-\beta] M(0, T, \alpha) \\
& +\frac{1}{2} \sigma_{3}^{2} \int_{0}^{T} M^{2}(u, T, \alpha) d u \\
& \left.+H\left(\sigma_{3}^{H}\right)^{2} \int_{0}^{T} M(u, T, \alpha) u^{2 H-1} d u\right\},
\end{aligned}
$$

so

$$
\begin{aligned}
& \frac{d Q^{\lambda}}{d Q} \mid \mathscr{F}_{0}=\frac{e^{-\int_{0}^{T} \lambda(u) d u}}{E\left[e^{-\int_{0}^{T} \lambda(u) d u} \mid \mathscr{F}_{0}\right]} \\
& \quad=\exp \left\{-\sigma_{3} \int_{0}^{T} M(u, T, \alpha) d W_{3}(u)\right.
\end{aligned}
$$

Using fractional Girsanov's theorem

$$
\begin{aligned}
W_{1}^{\lambda}(T)= & W_{1}(T)+\rho_{13} \sigma_{3} \int_{0}^{T} M(u, T, \alpha) d u, \\
W_{2}^{\lambda}(T)= & W_{2}(T)+\rho_{23} \sigma_{3} \int_{0}^{T} M(u, T, \alpha) d u, \\
W_{3}^{\lambda}(T)= & W_{3}(T)+\sigma_{3} \int_{0}^{T} M(u, T, \alpha) d u, \\
W_{1}^{H \lambda}(T)= & W_{1}^{H}(T) \\
& +2 H \rho_{13}^{H} \sigma_{3}^{H} \int_{0}^{T} M(u, T, \alpha) u^{2 H-1} d u, \\
W_{2}^{H \lambda}(T)= & W_{2}^{H}(T) \\
& +2 H \rho_{23}^{H} \sigma_{3}^{H} \int_{0}^{T} M(u, T, \alpha) u^{2 H-1} d u, \\
W_{3}^{H \lambda}(T)= & W_{3}^{H}(T) \\
& +2 H \sigma_{3}^{H} \int_{0}^{T} M(u, T, \alpha) u^{2 H-1} d u,
\end{aligned}
$$

according to Equation (14), therefore, we have the expression for $S_{T}$ and $V_{T}$ under measure $Q^{\lambda}$

$$
\begin{aligned}
S_{T} & =S_{0} \exp \left\{r T-\theta \lambda_{1} T-\frac{1}{2} \sigma_{1}{ }^{2} T+\int_{0}^{T} \sigma_{1} d W_{1}(u)\right. \\
& -\frac{1}{2}\left(\sigma_{1}^{H}\right)^{2} T^{2 H}+\int_{0}^{T} \sigma_{1}^{H} d W_{1}^{H}(u) \\
& \left.+\sum_{i=0}^{N_{T}} \ln \left(Y_{i}+1\right)\right\}=S_{0} \exp \left\{r T-\frac{1}{2} \sigma_{1}^{2} T\right. \\
& -\frac{1}{2}\left(\sigma_{1}^{H}\right)^{2} T^{2 H}+\int_{0}^{T} \sigma_{1} d W_{1}^{\lambda}(u) \\
& -\rho_{13} \sigma_{1} \sigma_{3} \int_{0}^{T} M(u, T, \alpha) d u+\int_{0}^{T} \sigma_{1}^{H} d W_{1}^{H \lambda}(u) \\
& -2 H \rho_{13}^{H} \sigma_{1}^{H} \sigma_{3}^{H} \int_{0}^{T} M(u, T, \alpha) u^{2 H-1} d u-\theta \lambda_{1} T \\
& \left.+\sum_{i=0}^{N_{T}} \ln \left(Y_{i}+1\right)\right\},
\end{aligned}
$$




$$
\begin{aligned}
V_{T} & =V_{0} \exp \left\{r T-\frac{1}{2} \sigma_{2}^{2} T+\int_{0}^{T} \sigma_{2} d W_{2}(u)\right. \\
& \left.-\frac{1}{2}\left(\sigma_{2}^{H}\right)^{2} T^{2 H}+\int_{0}^{T} \sigma_{2}^{H} d W_{2}^{H}(u)\right\}=V_{0} \exp \{r T \\
& -\frac{1}{2} \sigma_{2}^{2} T+\int_{0}^{T} \sigma_{2} d W_{2}^{\lambda}(u) \\
& -\rho_{23} \sigma_{2} \sigma_{3} \int_{0}^{T} M(u, T, \alpha) d u-\frac{1}{2}\left(\sigma_{2}^{H}\right)^{2} T^{2 H} \\
& +\int_{0}^{T} \sigma_{2}^{H} d W_{2}^{H \lambda}(u) \\
& -2 H \rho_{23}^{H} \sigma_{2}^{H} \sigma_{3}^{H} \int_{0}^{T} M(u, T, \alpha) u^{2 H-1} d u .
\end{aligned}
$$

Using Radon - Nikodym derivative

$$
I_{4}=E\left[e^{-r T-\int_{0}^{T} \lambda(u) d u} K I_{\left\{S_{T}>K, V_{T}>D\right\}} \mid \mathscr{F}_{0}\right]
$$

$$
\begin{aligned}
& =e^{-r T} K E\left[e^{-\int_{0}^{T} \lambda(u) d u} I_{\left\{S_{T}>K, V_{T}>D\right\}} \mid \mathscr{F}_{0}\right] \\
& =e^{-r T} K A(0, T) E^{\lambda}\left[I_{\left\{S_{T}>K, V_{T}>D\right\}} \mid \mathscr{F}_{0}\right],
\end{aligned}
$$

when $N_{T}=n$, we bring $S_{T}$ and $V_{T}$ into $I_{\left\{S_{T}>K, V_{T}>D\right\}}$

$$
\begin{aligned}
E^{\lambda} & {\left[I_{\left\{S_{T}>K, V_{T}>D\right\}} \mid \mathscr{F}_{0}\right] } \\
& =\sum_{n=0}^{\infty} \frac{\left(\lambda_{1} T\right)^{n}}{n !} e^{-\lambda_{1} T} N\left(d_{5}(n), d_{6}(n),\left(\rho_{12}, \rho_{12}^{H}\right)\right),
\end{aligned}
$$

so

$$
\begin{aligned}
I_{4} & =e^{-r T} K A(0, T) \\
& \cdot \sum_{n=0}^{\infty} \frac{\left(\lambda_{1} T\right)^{n}}{n !} e^{-\lambda_{1} T} N\left(d_{5}(n), d_{6}(n),\left(\rho_{12}, \rho_{12}^{H}\right)\right),
\end{aligned}
$$

where

$$
\begin{aligned}
d_{5}(n)= & \frac{\ln \left(S_{0} / K\right)+r T-(1 / 2) \sigma_{1}{ }^{2} T-\rho_{13} \sigma_{1} \sigma_{3} \int_{0}^{T} M(u, T, \alpha) d u-(1 / 2)\left(\sigma_{1}^{H}\right)^{2} T}{\sqrt{\sigma_{1}^{2} T+\left(\sigma_{1}^{H}\right)^{2} T^{2 H}+n \sigma_{J}^{2}}} \\
& +\frac{-2 H \rho_{13}^{H} \sigma_{1}^{H} \sigma_{3}^{H} \int_{0}^{T} M(u, T, \alpha) u^{2 H-1} d u-\theta \lambda_{1} T+n \mu_{J}}{\sqrt{\sigma_{1}^{2} T+\left(\sigma_{1}^{H}\right)^{2} T^{2 H}+n \sigma_{J}^{2}}} \\
= & d_{3}(n)-\frac{\sigma_{1}{ }^{2} T+(1 / 2)\left(\sigma_{1}^{H}\right)^{2}\left(T+T^{2 H}\right)+\left(\lambda_{1}-\lambda_{2}\right) \theta T}{\sqrt{\sigma_{1}{ }^{2} T+\left(\sigma_{1}^{H}\right)^{2} T^{2 H}+n \sigma_{J}^{2}}}, \\
d_{6}(n)= & \frac{\ln \left(V_{0} / D\right)+r T-(1 / 2) \sigma_{2}{ }^{2} T-\rho_{23} \sigma_{2} \sigma_{3} \int_{0}^{T} M(u, T, \alpha) d u}{\sqrt{\sigma_{2}{ }^{2} T+\left(\sigma_{2}^{H}\right)^{2} T^{2 H}}} \\
& +\frac{-(1 / 2)\left(\sigma_{2}^{H}\right)^{2} T^{2 H}-2 H \rho_{23}^{H} \sigma_{2}^{H} \sigma_{3}^{H} \int_{0}^{T} M(u, T, \alpha) u^{2 H-1} d u}{\sqrt{\sigma_{2}{ }^{2} T+\left(\sigma_{2}^{H}\right)^{2} T^{2 H}}}=d_{4}(n)-\frac{\rho_{12} \sigma_{1} \sigma_{2} T+\rho_{12}^{H} \sigma_{1}^{H} \sigma_{2}^{H} T^{2 H}}{\sqrt{\sigma_{2}{ }^{2} T+\left(\sigma_{2}^{H}\right)^{2} T^{2 H}}} .
\end{aligned}
$$

Then we calculate $I_{3}$, let measure $Q^{S}$ in

$$
\frac{d Q^{S}}{d Q^{\lambda}} \mid \mathscr{F}_{0}=\frac{S_{T}}{E^{\lambda}\left[S_{T} \mid \mathscr{F}_{0}\right]},
$$

let

$$
X(0, T)=E^{\lambda}\left[S_{T} \mid \mathscr{F}_{0}\right],
$$

under the measure $Q^{\lambda}$, we bring Equation (A.14) into Equation (A.21)

$$
\begin{gathered}
X(0, T)=S_{0} \exp \left\{r T-\rho_{13} \sigma_{1} \sigma_{3} \int_{0}^{T} M(u, T, \alpha) d u\right. \\
\left.-2 H \rho_{13}^{H} \sigma_{1}^{H} \sigma_{3}^{H} \int_{0}^{T} M(u, T, \alpha) u^{2 H-1} d u\right\},
\end{gathered}
$$

hence

$$
\begin{aligned}
& \frac{d Q^{S}}{d Q^{\lambda}} \mid \mathscr{F}_{0}=\exp \left\{-\frac{1}{2} \sigma_{1}{ }^{2} T+\int_{0}^{T} \sigma_{1} d W_{1}^{\lambda}(u)\right. \\
& -\frac{1}{2}\left(\sigma_{1}^{H}\right)^{2} T^{2 H}+\int_{0}^{T} \sigma_{1}^{H} d W_{1}^{H \lambda}(u)-\theta \lambda_{1} T \\
& \left.+\sum_{i=0}^{N_{T}} \ln \left(Y_{i}+1\right)\right\} .
\end{aligned}
$$

Using fractional Girsanov's theorem, we derive

$$
\begin{aligned}
& W_{1}^{S}(T)=W_{1}^{\lambda}(T)-\sigma_{1} T, \\
& W_{2}^{S}(T)=W_{2}^{\lambda}(T)-\rho_{12} \sigma_{1} T,
\end{aligned}
$$




$$
\begin{aligned}
& W_{1}^{H S}(T)=W_{1}^{H \lambda}(T)-\sigma_{1}^{H} T^{2 H}, \\
& W_{2}^{H S}(T)=W_{2}^{H \lambda}(T)-\rho_{12}^{H} \sigma_{1}^{H} T^{2 H},
\end{aligned}
$$$$
\left.-2 H \rho_{23}^{H} \sigma_{2}^{H} \sigma_{3}^{H} \int_{0}^{T} M(u, T, \alpha) u^{2 H-1} d u\right\} .
$$

under measure $Q^{S}$, we have

$$
\begin{aligned}
S_{T} & =S_{0} \exp \left\{r T+\frac{1}{2} \sigma_{1}^{2} T+\int_{0}^{T} \sigma_{1} d W_{1}^{s}(u)\right. \\
& -\rho_{13} \sigma_{1} \sigma_{3} \int_{0}^{T} M(u, T, \alpha) d u+\frac{1}{2}\left(\sigma_{1}^{H}\right)^{2} T^{2 H} \\
& +\int_{0}^{T} \sigma_{1}^{H} d W_{1}^{H S}(u) \\
& -2 H \rho_{13}^{H} \sigma_{1}^{H} \sigma_{3}^{H} \int_{0}^{T} M(u, T, \alpha) u^{2 H-1} d u-\theta \lambda_{2} T \\
& \left.+\sum_{i=0}^{N_{T}} \ln \left(Y_{i}+1\right)\right\}, \\
V_{T} & =V_{0} \exp \left\{r T-\frac{1}{2} \sigma_{2}^{2} T+\int_{0}^{T} \sigma_{2} d W_{2}^{S}(u)\right. \\
& +\rho_{12} \sigma_{1} \sigma_{2} T-\rho_{23} \sigma_{2} \sigma_{3} \int_{0}^{T} M(u, T, \alpha) d u \\
& -\frac{1}{2}\left(\sigma_{2}^{H}\right)^{2} T^{2 H}+\int_{0}^{T} \sigma_{2}^{H} d W_{2}^{H S}(u)+\rho_{12}^{H} \sigma_{1}^{H} \sigma_{2}^{H} T
\end{aligned}
$$

Using Radon - Nikodym derivative, we have

$$
\begin{aligned}
I_{3} & =E\left[e^{-r T-\int_{0}^{T} \lambda(u) d u} S_{T} I_{\left\{S_{T}>K, V_{T}>D\right\}} \mid \mathscr{F}_{0}\right] \\
& =e^{-r T} E\left[e^{-\int_{0}^{T} \lambda(u) d u} S_{T} I_{\left\{S_{T}>K, V_{T}>D\right\}} \mid \mathscr{F}_{0}\right] \\
& =e^{-r T} A(0, T) E^{\lambda}\left[S_{T} I_{\left\{S_{T}>K, V_{T}>D\right\}} \mid \mathscr{F}_{0}\right] \\
& =e^{-r T} A(0, T) X(0, T) E^{S}\left[I_{\left\{S_{T}>K, V_{T}>D\right\}} \mid \mathscr{F}_{0}\right],
\end{aligned}
$$

when $N_{T}=n$, we bring $S_{T}$ and $V_{T}$ into $I_{\left\{S_{T}>K, V_{T}>D\right\}}$

$$
\begin{aligned}
E^{S} & {\left[I_{\left\{S_{T}>K, V_{T}>D\right\}} \mid \mathscr{F}_{0}\right] } \\
& =\sum_{n=0}^{\infty} \frac{\left(\lambda_{2} T\right)^{n}}{n !} e^{-\lambda_{2} T} N\left(d_{3}(n), d_{4}(n),\left(\rho_{12}, \rho_{12}^{H}\right)\right),
\end{aligned}
$$

so

$$
\begin{aligned}
I_{3}= & e^{-r T} A(0, T) X(0, T) \\
& \cdot \sum_{n=0}^{\infty} \frac{\left(\lambda_{2} T\right)^{n}}{n !} e^{-\lambda_{2} T} N\left(d_{3}(n), d_{4}(n),\left(\rho_{12}, \rho_{12}^{H}\right)\right),
\end{aligned}
$$

where

$$
\begin{gathered}
d_{3}(n)=\frac{\ln \left(S_{0} / K\right)+r T+(1 / 2) \sigma_{1}^{2} T-\rho_{13} \sigma_{1} \sigma_{3} \int_{0}^{T} M(u, T, \alpha) d u+(1 / 2)\left(\sigma_{1}^{H}\right)^{2} T^{2 H}}{\sqrt{\sigma_{1}^{2} T+\left(\sigma_{1}^{H}\right)^{2} T^{2 H}+n \sigma_{J}^{2}}} \\
+\frac{-2 H \rho_{13}^{H} \sigma_{1}^{H} \sigma_{3}^{H} \int_{0}^{T} M(u, T, \alpha) u^{2 H-1} d u-\theta \lambda_{2} T+n \mu_{J}}{\sqrt{\sigma_{1}^{2} T+\left(\sigma_{1}^{H}\right)^{2} T^{2 H}+n \sigma_{J}^{2}}}, \\
d_{4}(n)=\frac{\ln \left(V_{0} / D\right)+r T-(1 / 2) \sigma_{2}^{2} T+\rho_{12} \sigma_{1} \sigma_{2} T-\rho_{23} \sigma_{2} \sigma_{3} \int_{0}^{T} M(u, T, \alpha) d u-(1 / 2)\left(\sigma_{2}^{H}\right)^{2} T^{2 H}}{\sqrt{\sigma_{2}^{2} T+\left(\sigma_{2}^{H}\right)^{2} T^{2 H}}} \\
+\frac{\rho_{12}^{H} \sigma_{1}^{H} \sigma_{2}^{H} T^{2 H}-2 H \rho_{23}^{H} \sigma_{2}^{H} \sigma_{3}^{H} \int_{0}^{T} M(u, T, \alpha) u^{2 H-1} d u}{\sqrt{\sigma_{2}^{2} T+\left(\sigma_{2}^{H}\right)^{2} T^{2 H}}} .
\end{gathered}
$$

so

$$
I I=I_{3}-I_{4}
$$

$$
\begin{aligned}
& =E\left[e^{-r T-\int_{0}^{T} \lambda(u) d u} \frac{1-w}{D} V_{T}\left(S_{T}-K\right)^{+} I_{\left\{V_{T}>D\right\}} \mid \mathscr{F}_{0}\right] \\
& =I_{5}-I_{6},
\end{aligned}
$$

\section{B. Derivation Process of III}

Deduced III. We define

where

$$
I_{5}=E\left[e^{-r T-\int_{0}^{T} \lambda(u) d u} \frac{1-w}{D} S_{T} V_{T} I_{\left\{S_{T}>K, V_{T}>D\right\}} \mid \mathscr{F}_{0}\right],
$$




$$
I_{6}=E\left[e^{-r T-\int_{0}^{T} \lambda(u) d u} \frac{1-w}{D} K V_{T} I_{\left\{S_{T}>K, V_{T}>D\right\}} \mid \mathscr{F}_{0}\right] .
$$

Firstly we calculate $I_{6}$. Let new measure $Q^{V}$ in

$$
\frac{d Q^{V}}{d Q^{\lambda}} \mid \mathscr{F}_{0}=\frac{V_{T}}{E^{\lambda}\left[V_{T} \mid \mathscr{F}_{0}\right]},
$$

let

$$
Y(0, T)=E^{\lambda}\left[V_{T} \mid \mathscr{F}_{0}\right],
$$

under measure $Q^{\lambda}$, we have

$$
\begin{aligned}
V_{T} & =V_{0} \exp \left\{r T-\frac{1}{2} \sigma_{2}^{2} T+\int_{0}^{T} \sigma_{2} d W_{2}^{\lambda}(u)\right. \\
& -\rho_{23} \sigma_{2} \sigma_{3} \int_{0}^{T} M(u, T, \alpha) d u-\frac{1}{2}\left(\sigma_{2}^{H}\right)^{2} T^{2 H} \\
& +\int_{0}^{T} \sigma_{2}^{H} d W_{2}^{H \lambda}(u)-2 H \rho_{23}^{H} \sigma_{2}^{H} \sigma_{3}^{H} \\
& \left.\cdot \int_{0}^{T} M(u, T, \alpha) u^{2 H-1} d u\right\},
\end{aligned}
$$

then we have

$$
\begin{gathered}
Y(0, T)=V_{0} \exp \left\{r T-\rho_{23} \sigma_{2} \sigma_{3} \int_{0}^{T} M(u, T, \alpha) d u\right. \\
\left.-2 H \rho_{23}^{H} \sigma_{2}^{H} \sigma_{3}^{H} \int_{0}^{T} M(u, T, \alpha) u^{2 H-1} d u\right\},
\end{gathered}
$$

so

$$
\begin{gathered}
\frac{d Q^{V}}{d Q^{\lambda}} \mid \mathscr{F}_{0}=\exp \left\{-\frac{1}{2} \sigma_{2}^{2} T+\int_{0}^{T} \sigma_{2} d W_{2}^{\lambda}(u)\right. \\
\left.-\frac{1}{2}\left(\sigma_{2}^{H}\right)^{2} T^{2 H}+\int_{0}^{T} \sigma_{2}^{H} d W_{2}^{H \lambda}(u)\right\} .
\end{gathered}
$$

Using fractional Girsanov's theorem

$$
\begin{aligned}
W_{1}^{V}(T) & =W_{1}^{\lambda}(T)-\rho_{12} \sigma_{2} T, \\
W_{2}^{V}(T) & =W_{2}^{\lambda}(T)-\sigma_{2} T, \\
W_{1}^{H V}(T) & =W_{1}^{H \lambda}(T)-\rho_{12}^{H} \sigma_{2}^{H} T^{2 H}, \\
W_{2}^{H V}(T) & =W_{2}^{H \lambda}(T)-\sigma_{2}^{H} T^{2 H},
\end{aligned}
$$

since under measure $Q^{V}$

$$
S_{T}=S_{0} \exp \left\{r T-\frac{1}{2} \sigma_{1}^{2} T+\int_{0}^{T} \sigma_{1} d W_{1}^{V}(u)\right.
$$

$$
\begin{aligned}
& -\rho_{13} \sigma_{1} \sigma_{3} \int_{0}^{T} M(u, T, \alpha) d u+\rho_{12} \sigma_{1} \sigma_{2} T \\
& -\frac{1}{2}\left(\sigma_{1}^{H}\right)^{2} T^{2 H}+\int_{0}^{T} \sigma_{1}^{H} d W_{1}^{H V}(u) \\
& -2 H \rho_{13}^{H} \sigma_{1}^{H} \sigma_{3}^{H} \int_{0}^{T} M(u, T, \alpha) u^{2 H-1} d u \\
& \left.+\rho_{12}^{H} \sigma_{1}^{H} \sigma_{2}^{H} T^{2 H}-\theta \lambda_{1} T+\sum_{i=0}^{N_{T}} \ln \left(Y_{i}+1\right)\right\},
\end{aligned}
$$

$$
\begin{aligned}
V_{T} & =V_{0} \exp \left\{r T+\frac{1}{2} \sigma_{2}^{2} T+\int_{0}^{T} \sigma_{2} d W_{2}^{V}(u)\right. \\
& -\rho_{23} \sigma_{2} \sigma_{3} \int_{0}^{T} M(u, T, \alpha) d u+\frac{1}{2}\left(\sigma_{2}^{H}\right)^{2} T^{2 H} \\
& +\int_{0}^{T} \sigma_{2}^{H} d W_{2}^{H V}(u) \\
& \left.-2 H \rho_{23}^{H} \sigma_{2}^{H} \sigma_{3}^{H} \int_{0}^{T} M(u, T, \alpha) u^{2 H-1} d u\right\},
\end{aligned}
$$

using Radon - Nikodym derivative, we obtain

$$
\begin{aligned}
I_{6} & =E\left[e^{-r T-\int_{0}^{T} \lambda(u) d u} \frac{1-w}{D} K V_{T} I_{\left\{S_{T}>K, V_{T}>D\right\}} \mid \mathscr{F}_{0}\right] \\
& =e^{-r T} \frac{1-w}{D} \\
& \cdot K E\left[e^{-\int_{0}^{T} \lambda(u) d u} V_{T} I_{\left\{S_{T}>K, V_{T}>D\right\}} \mid \mathscr{F}_{0}\right]=e^{-r T} \\
& \cdot \frac{1-w}{D} K A(0, T) E^{\lambda}\left[V_{T} I_{\left\{S_{T}>K, V_{T}>D\right\}} \mid \mathscr{F}_{0}\right] \\
& =e^{-r T} \frac{1-w}{D} K A(0, T) Y(0, T) \\
& \cdot E^{V}\left[I_{\left\{S_{T}>K, V_{T}>D\right\}} \mid \mathscr{F}_{0}\right],
\end{aligned}
$$

when $N_{T}=n$, we bring $S_{T}$ and $V_{T}$ into

$$
\begin{aligned}
E^{V} & {\left[I_{\left\{S_{T}>K, V_{T}>D\right\}} \mid \mathscr{F}_{0}\right] } \\
= & \sum_{n=0}^{\infty} \frac{\left(\lambda_{1} T\right)^{n}}{n !} e^{-\lambda_{1} T} N\left(d_{9}(n), d_{10}(n),\left(\rho_{12}, \rho_{12}^{H}\right)\right),
\end{aligned}
$$

so

$$
\begin{aligned}
I_{6} & =e^{-r T} \frac{1-w}{D} K A(0, T) Y(0, T) \\
& \cdot \sum_{n=0}^{\infty} \frac{\left(\lambda_{1} T\right)^{n}}{n !} e^{-\lambda_{1} T} N\left(d_{9}(n), d_{10}(n),\left(\rho_{12}, \rho_{12}^{H}\right)\right),
\end{aligned}
$$

where

$$
d_{9}(n)=\frac{\ln \left(S_{0} / K\right)+r T-(1 / 2) \sigma_{1}^{2} T-\rho_{13} \sigma_{1} \sigma_{3} \int_{0}^{T} M(u, T, \alpha) d u+\rho_{12} \sigma_{1} \sigma_{2} T-(1 / 2)\left(\sigma_{1}^{H}\right)^{2} T^{2 H}}{\sqrt{\sigma_{1}^{2} T+\left(\sigma_{1}^{H}\right)^{2} T^{2 H}+n \sigma_{J}^{2}}}
$$




$$
\begin{gathered}
+\frac{-2 H \rho_{13}^{H} \sigma_{1}^{H} \sigma_{3}^{H} \int_{0}^{T} M(u, T, \alpha) u^{2 H-1} d u+\rho_{12}^{H} \sigma_{1}^{H} \sigma_{2}^{H} T^{2 H}-\theta \lambda_{1} T+n \mu_{J}}{\sqrt{\sigma_{1}^{2} T+\left(\sigma_{1}^{H}\right)^{2} T^{2 H}+n \sigma_{J}^{2}}} \\
=d_{3}(n)-\frac{\sigma_{1}^{2} T+\left(\sigma_{1}^{H}\right)^{2} T^{2 H}-\rho_{12} \sigma_{1} \sigma_{2} T-\left(\lambda_{2}-\lambda_{1}\right) \theta T}{\sqrt{\sigma_{1}^{2} T+\left(\sigma_{1}^{H}\right)^{2} T^{2 H}+n \sigma_{J}^{2}}}, \\
d_{10}(n)= \\
+\frac{\ln \left(V_{0} / D\right)+r T+(1 / 2) \sigma_{2}^{2} T-\rho_{23} \sigma_{2} \sigma_{3} \int_{0}^{T} M(u, T, \alpha) d u}{\sqrt{\sigma_{2}^{2} T+\left(\sigma_{2}^{H}\right)^{2} T^{2 H}}} \\
+\frac{(1 / 2)\left(\sigma_{2}^{H}\right)^{2} T^{2 H}-2 H \rho_{23}^{H} \sigma_{2}^{H} \sigma_{3}^{H} \int_{0}^{T} M(u, T, \alpha) u^{2 H-1} d u}{\sqrt{\sigma_{2}^{2} T+\left(\sigma_{2}^{H}\right)^{2} T^{2 H}}}=d_{6}(n)+\sqrt{\sigma_{2}^{2} T+\left(\sigma_{2}^{H}\right)^{2} T^{2 H}} .
\end{gathered}
$$

Then we will calculate $I_{5}$. Let measure $Q^{S V}$ in

$$
\frac{d Q^{S V}}{d Q^{\lambda}} \mid \mathscr{F}_{0}=\frac{S_{T} V_{T}}{E^{\lambda}\left[S_{T} V_{T} \mid \mathscr{F}_{0}\right]},
$$

let

$$
Z(0, T)=E^{\lambda}\left[S_{T} V_{T} \mid \mathscr{F}_{0}\right],
$$

under measure $Q^{\lambda}$

$$
\begin{aligned}
S_{T} & =S_{0} \exp \left\{r T-\frac{1}{2} \sigma_{1}^{2} T+\int_{0}^{T} \sigma_{1} d W_{1}^{\lambda}(u)\right. \\
& -\rho_{13} \sigma_{1} \sigma_{3} \int_{0}^{T} M(u, T, \alpha) d u-\frac{1}{2}\left(\sigma_{1}^{H}\right)^{2} T^{2 H} \\
& +\int_{0}^{T} \sigma_{1}^{H} d W_{1}^{H \lambda}(u) \\
& -2 H \rho_{13}^{H} \sigma_{1}^{H} \sigma_{3}^{H} \int_{0}^{T} M(u, T, \alpha) u^{2 H-1} d u-\theta \lambda_{1} T \\
& \left.+\sum_{i=0}^{N_{T}} \ln \left(Y_{i}+1\right)\right\}, \\
V_{T} & =V_{0} \exp \left\{r T-\frac{1}{2} \sigma_{2}^{2} T+\int_{0}^{T} \sigma_{2} d W_{2}^{\lambda}(u)\right. \\
& -\rho_{23} \sigma_{2} \sigma_{3} \int_{0}^{T} M(u, T, \alpha) d u-\frac{1}{2}\left(\sigma_{2}^{H}\right)^{2} T^{2 H} \\
& +\int_{0}^{T} \sigma_{2}^{H} d W_{2}^{H \lambda}(u) \\
& \left.-2 H \rho_{23}^{H} \sigma_{2}^{H} \sigma_{3}^{H} \int_{0}^{T} M(u, T, \alpha) u^{2 H-1} d u\right\},
\end{aligned}
$$

we have

$$
\begin{aligned}
& Z(0, T)=S_{0} V_{0} \exp \left\{2 r T-\left(\rho_{13} \sigma_{1}+\rho_{23} \sigma_{2}\right)\right. \\
& \cdot \sigma_{3} \int_{0}^{T} M(u, T, \alpha) d u+\rho_{12} \sigma_{1} \sigma_{2} \\
& -2 H\left(\rho_{13}^{H} \sigma_{1}^{H}+\rho_{23}^{H} \sigma_{2}^{H}\right) \\
& \left.\cdot \sigma_{3}^{H} \int_{0}^{T} M(u, T, \alpha) u^{2 H-1} d u+\rho_{12}^{H} \sigma_{1}^{H} \sigma_{2}^{H}\right\},
\end{aligned}
$$

so

$$
\begin{aligned}
& \frac{d Q^{S V}}{d Q^{\lambda}} \mid \mathscr{F}_{0}=\exp \left\{-\frac{1}{2}\left(\sigma_{1}{ }^{2}+\sigma_{2}{ }^{2}+\rho_{12} \sigma_{1} \sigma_{2}\right) T\right. \\
& \quad+\int_{0}^{T} \sigma_{1} d W_{1}^{\lambda}(u)+\int_{0}^{T} \sigma_{2} d W_{2}^{\lambda}(u) \\
& \quad-\frac{1}{2}\left(\left(\sigma_{1}^{H}\right)^{2}+\left(\sigma_{2}^{H}\right)^{2}+\rho_{12}^{H} \sigma_{1}^{H} \sigma_{2}^{H}\right) T^{2 H} \\
& \quad+\int_{0}^{T} \sigma_{1}^{H} d W_{1}^{H \lambda}(u)+\int_{0}^{T} \sigma_{2}^{H} d W_{2}^{H \lambda}(u)-\theta \lambda_{1} T \\
& \left.\quad+\sum_{i=0}^{N_{T}} \ln \left(Y_{i}+1\right)\right\} .
\end{aligned}
$$

Using fractional Girsanov's theorem, we have

$$
\begin{aligned}
W_{1}^{S V}(T) & =W_{1}^{\lambda}(T)-\sigma_{1} T-\rho_{12} \sigma_{2} T, \\
W_{2}^{S V}(T) & =W_{2}^{\lambda}(T)-\sigma_{2} T-\rho_{12} \sigma_{1} T, \\
W_{1}^{H S V}(T) & =W_{1}^{H \lambda}(T)-\sigma_{1}^{H} T^{2 H}-\rho_{12}^{H} \sigma_{2}^{H} T^{2 H}, \\
W_{2}^{H S V}(T) & =W_{2}^{H \lambda}(T)-\sigma_{2}^{H} T^{2 H}-\rho_{12}^{H} \sigma_{1}^{H} T^{2 H},
\end{aligned}
$$


we have the expression for $S_{T}$ and $V_{T}$ under measure $Q^{S V}$

$$
\begin{aligned}
S_{T} & =S_{0} \exp \left\{r T+\frac{1}{2} \sigma_{1}{ }^{2} T+\int_{0}^{T} \sigma_{1} d W_{1}^{S V}(u)\right. \\
& -\rho_{13} \sigma_{1} \sigma_{3} \int_{0}^{T} M(u, T, \alpha) d u+\rho_{12} \sigma_{1} \sigma_{2} T \\
& +\int_{0}^{T} \sigma_{1}^{H} d W_{1}^{H S V}(u) \\
& -2 H \rho_{13}^{H} \sigma_{1}^{H} \sigma_{3}^{H} \int_{0}^{T} M(u, T, \alpha) u^{2 H-1} d u \\
& +\rho_{12}^{H} \sigma_{1}^{H} \sigma_{2}^{H} T^{2 H}+\frac{1}{2}\left(\sigma_{1}^{H}\right)^{2} T^{2 H}-\theta \lambda_{2} T \\
& \left.+\frac{1}{2}\left(\sigma_{2}^{H}\right)^{2} T^{2 H}+\rho_{12}^{H} \sigma_{1}^{H} \sigma_{2}^{H} T^{2 H}\right\}, \\
& \left.+\sum_{i=0} N_{T} \ln \left(Y_{i}+1\right)\right\}, \\
& +\int_{0}^{T} \sigma_{2}^{H} d W_{2}^{H S V}(u) \\
& -\rho_{23} \sigma_{2} \sigma_{3} \int_{0}^{T} M(u, T, \alpha) d u+\rho_{12} \sigma_{1} \sigma_{2} T \\
V_{T} & =V_{0} \rho_{23}^{H} \sigma_{2}^{H} \sigma_{3}^{H} \int_{0}^{T} M(u, T, \alpha) u^{2 H-1} d u \\
& \exp ^{2 H}+\frac{1}{2} \sigma_{2}{ }^{2} T+\int_{0}^{T} \sigma_{2} d W_{2}^{S V}(u)
\end{aligned}
$$

$$
\begin{aligned}
I_{5}= & e^{-r T} \frac{1-w}{D} k A(0, T) Z(0, T) \\
& \cdot \sum_{n=0}^{\infty} \frac{\left(\lambda_{2} T\right)^{n}}{n !} e^{-\lambda_{2} T} N\left(d_{7}(n), d_{8}(n),\left(\rho_{12}, \rho_{12}^{H}\right)\right),
\end{aligned}
$$

when $N_{T}=n$, we bring $S_{T}$ and $V_{T}$ into

$$
\begin{aligned}
E^{S V} & {\left[I_{\left\{S_{T}>K, V_{T}>D\right\}} \mid \mathscr{F}_{0}\right] } \\
& =\sum_{n=0}^{\infty} \frac{\left(\lambda_{2} T\right)^{n}}{n !} e^{-\lambda_{2} T} N\left(d_{7}(n), d_{8}(n),\left(\rho_{12}, \rho_{12}^{H}\right)\right),
\end{aligned}
$$

where

$$
\begin{aligned}
d_{7}(n)= & \frac{\ln \left(S_{0} / K\right)+r T+(1 / 2) \sigma_{1}^{2} T-\rho_{13} \sigma_{1} \sigma_{3} \int_{0}^{T} M(u, T, \alpha) d u+\rho_{12} \sigma_{1} \sigma_{2} T+(1 / 2)\left(\sigma_{1}^{H}\right)^{2} T^{2 H}}{\sqrt{\sigma_{1}^{2} T+\left(\sigma_{1}^{H}\right)^{2} T^{2 H}+n \sigma_{J}^{2}}} \\
& +\frac{-2 H \rho_{13}^{H} \sigma_{1}^{H} \sigma_{3}^{H} \int_{0}^{T} M(u, T, \alpha) u^{2 H-1} d u+\rho_{12}^{H} \sigma_{1}^{H} \sigma_{2}^{H} T^{2 H}-\theta \lambda_{2} T+n \mu_{J}}{\sqrt{\sigma_{1}^{2} T+\left(\sigma_{1}^{H}\right)^{2} T^{2 H}+n \sigma_{J}^{2}}} \\
= & d_{3}(n)+\frac{\rho_{12} \sigma_{1} \sigma_{2} T+\rho_{12}^{H} \sigma_{1}^{H} \sigma_{2}^{H} T^{2 H}}{\sqrt{\sigma_{1}^{2} T+\left(\sigma_{1}^{H}\right)^{2} T^{2 H}+n \sigma_{J}^{2}}}, \\
d_{8}(n)= & \frac{\ln \left(V_{0} / D\right)+r T+(1 / 2) \sigma_{2}^{2} T+\rho_{12} \sigma_{1} \sigma_{2} T-\rho_{23} \sigma_{2} \sigma_{3} \int_{0}^{T} M(u, T, \alpha) d u}{\sqrt{\sigma_{2}^{2} T+\left(\sigma_{2}^{H}\right)^{2} T^{2 H}}} \\
& +\frac{(1 / 2)\left(\sigma_{2}^{H}\right)^{2} T^{2 H}+\rho_{12}^{H} \sigma_{1}^{H} \sigma_{2}^{H}-2 H \rho_{23}^{H} \sigma_{2}^{H} \sigma_{3}^{H} \int_{0}^{T} M(u, T, \alpha) u^{2 H-1} d u}{\sqrt{\sigma_{2}^{2} T+\left(\sigma_{2}^{H}\right)^{2} T^{2 H}}}=d_{4}(n)+\sqrt{\sigma_{2}^{2} T+\left(\sigma_{2}^{H}\right)^{2} T^{2 H}} .
\end{aligned}
$$


so

$$
I I I=I_{5}-I_{6}
$$

The proof of the theorem is completed.

\section{Data Availability}

The data used to support the findings of this study are available from the corresponding author upon request.

\section{Conflicts of Interest}

The authors declare that there are no conflicts of interest regarding the publication of this paper.

\section{Acknowledgments}

This work is supported by the National Natural Science Foundation of China (no. 61304088) and the Fundamental Research Funds for the Central Universities (no. 2013QNA37).

\section{References}

[1] R. C. Merton, "On the pricing of corporate debt: the risk structure of interest rates," The Journal of Finance, vol. 29, no. 2, pp. 449-470, 1974.

[2] F. Black and J. C. Cox, "Valuing corporate securities: some effects of bond indenture provisions," The Journal of Finance, vol. 31, no. 2, pp. 351-367, 1976.

[3] H. Johnson and R. Stulz, "The pricing of options with default ris," The Journal of Finance, vol. 42, no. 2, pp. 267-280, 1987.

[4] P. Klein, "Pricing black-scholes options with correlated credit risk, Journal of Banking \& Finance, vol. 20, no. 7, pp. 1211-1229, 1996.

[5] D. B. Madan and H. Unal, "Pricing the risks of default," Review of Derivatives Research, vol. 2, no. 2-3, pp. 121-160, 1998.

[6] B. J. Lobo, "Jump risk in the U.S. stock market: Evidence using political information," Review of Financial Economics, vol. 8, no. 2, pp. 149-163, 1999.

[7] C. Zhou, "The term structure of credit spreads with jump risk," Journal of Banking \& Finance, vol. 25, no. 11, pp. 2015-2040, 2001.

[8] M. Ammann, Credit Risk Valuation: Methods, Models and Applications, Springer Finance, Springer, Berlin, Germany, 2nd edition, 2002.

[9] L.-F. Chang and M.-W. Hung, "Valuation of vulnerable American options with correlated credit risk," Review of Derivatives Research, vol. 9, no. 2, pp. 137-165, 2006.

[10] L. Cathcart and L. El-Jahel, "Semi-analytical pricing of defaultable bonds in a signaling jump-default model," Journal of Computational Finance, vol. 6, no. 3, pp. 91-108, 2003.

[11] R. A. Jarrow and S. M. Turnbull, "Pricing derivatives on financial securities subject to credit risk," Journal of Finance, vol. 50, pp. 53-85, 1995.

[12] L. Cathcart and L. El-Jahel, "Pricing defaultable bonds: a middle-way approach between structural and reduced-form models," Quantitative Finance, vol. 6, no. 3, pp. 243-253, 2006.
[13] L. V. Ballestra and G. Pacelli, "Valuing risky debt: a new model combining structural information with the reducedform approach," Insurance: Mathematics \& Economics, vol. 55, pp. 261-271, 2014.

[14] L. V. Ballestra, G. Pacelli, and D. Radi, "Computing the survival probability in the Madan-Unal credit risk model: application to the CDS market," Quantitative Finance, vol. 17, no. 2, pp. 299313, 2017.

[15] T. Sottinen and E. Valkeila, Fractional Brownian Motion as a model in finance, 2001.

[16] Y. Hu and B. Øksendal, "Fractional white noise calculus and applications to finance," Infinite Dimensional Analysis, Quantum Probability and Related Topics, vol. 6, no. 1, pp. 1-32, 2003.

[17] B. Øksendal, Fractional Brownian Motion, University of Oslo, Oslo, Norway, 2004.

[18] T. Björk and H. Hult, "A note on Wick products and the fractional Black-Scholes model," Finance and Stochastics, vol. 9, no. 2, pp. 197-209, 2005.

[19] C. Patrick, "Mixed fractional brownian motion," Bernoulli, vol. 7, no. 6, pp. 913-934, 2001.

[20] T. Androshchuk and Y. Mishura, "Mixed Brownian-fractional Brownian model: absence of arbitrage and related topics," Stochastics. An International Journal of Probability and Stochastic Processes, vol. 78, no. 5, pp. 281-300, 2006.

[21] X.-T. Wang, E.-H. Zhu, M.-M. Tang, and H.-G. Yan, "Scaling and long-range dependence in option pricing II: pricing European option with transaction costs under the mixed Brownianfractional Brownian model," Physica A: Statistical Mechanics and its Applications, vol. 389, no. 3, pp. 445-451, 2010.

[22] L. V. Ballestra, G. Pacelli, and D. Radi, "A very efficient approach for pricing barrier options on an underlying described by the mixed fractional Brownian motion," Chaos, Solitons \& Fractals, vol. 87, pp. 240-248, 2016.

[23] C. Necula, "Option pricing in a fractional Brownian motion environment," Advances in Economic and Financial Research, vol. 2, no. 3, pp. 259-273, 2004.

[24] W. L. Huang, X. X. Tao, and S. H. Li, "Pricing formulae for European options under the fractional Vasicek interest rate model," Acta Mathematica Sinica, vol. 55, no. 2, pp. 219-230, 2012.

[25] X. Su and W. Wang, "Pricing options with credit risk in a reduced form model," Journal of the Korean Statistical Society, vol. 41, no. 4, pp. 437-444, 2012.

[26] J. Li and S. Ma, "Pricing Options with Credit Risk in Markovian Regime-Switching Markets," Journal of Applied Mathematics, vol. 2013, Article ID 621371, pp. 561-575, 2013.

[27] C. Wang, S. W. Zhou, and J. Y. Yang, “The pricing of vulnerable options in a fractional brownian motion environment," Discrete Dynamics in Nature and Society, vol. 2015, Article ID 579213, pp. 1-10, 2015.

[28] Y.-d. Sun, Y.-m. Shi, and X. Gu, "Barrier option pricing under the Vasicek model of the short rate," Journal of Applied Mathematics \& Informatics, vol. 29, no. 5-6, pp. 1501-1509, 2011.

[29] D. Lando, "On cox processes and credit risky securities," Review of Derivatives Research, vol. 2, no. 2-3, pp. 99-120, 1998.

[30] R. A. Jarrow and F. Yu, "Counterparty risk and the pricing of defaultable securities," Journal of Finance, vol. 56, no. 5, pp. 1765-1799, 2001. 


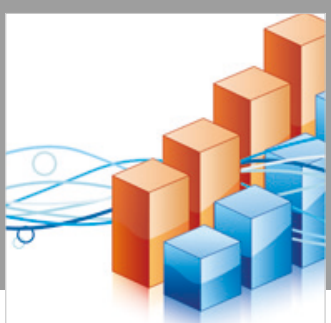

Advances in

Operations Research

\section{-n-m}
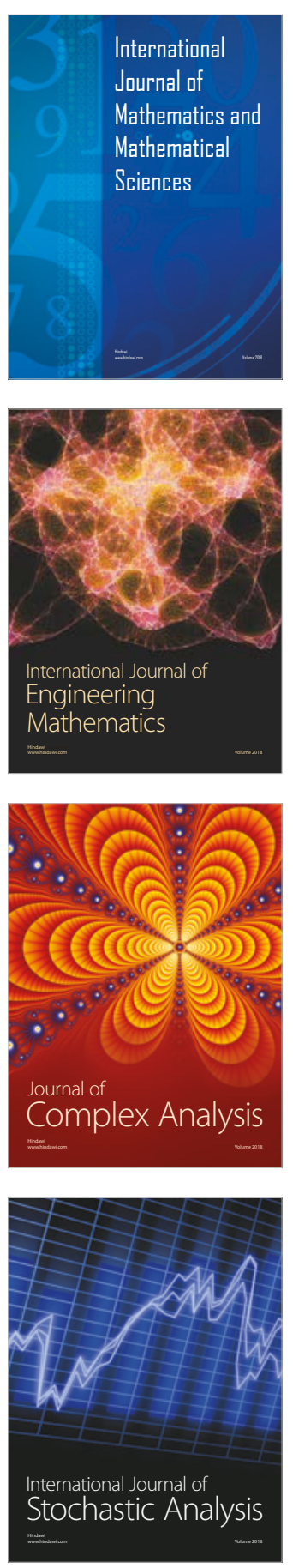
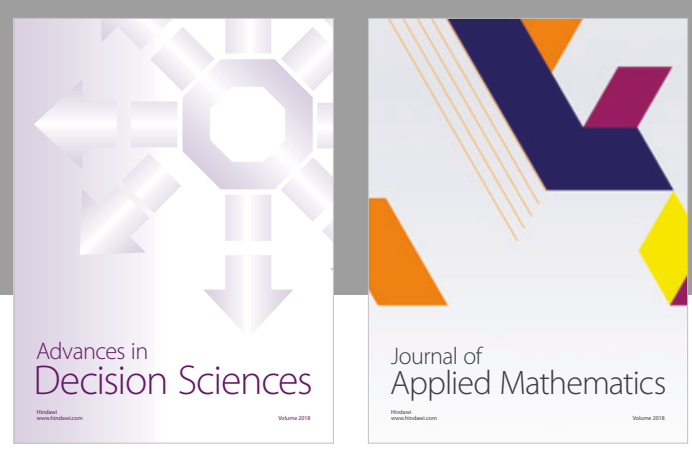

Journal of

Applied Mathematics
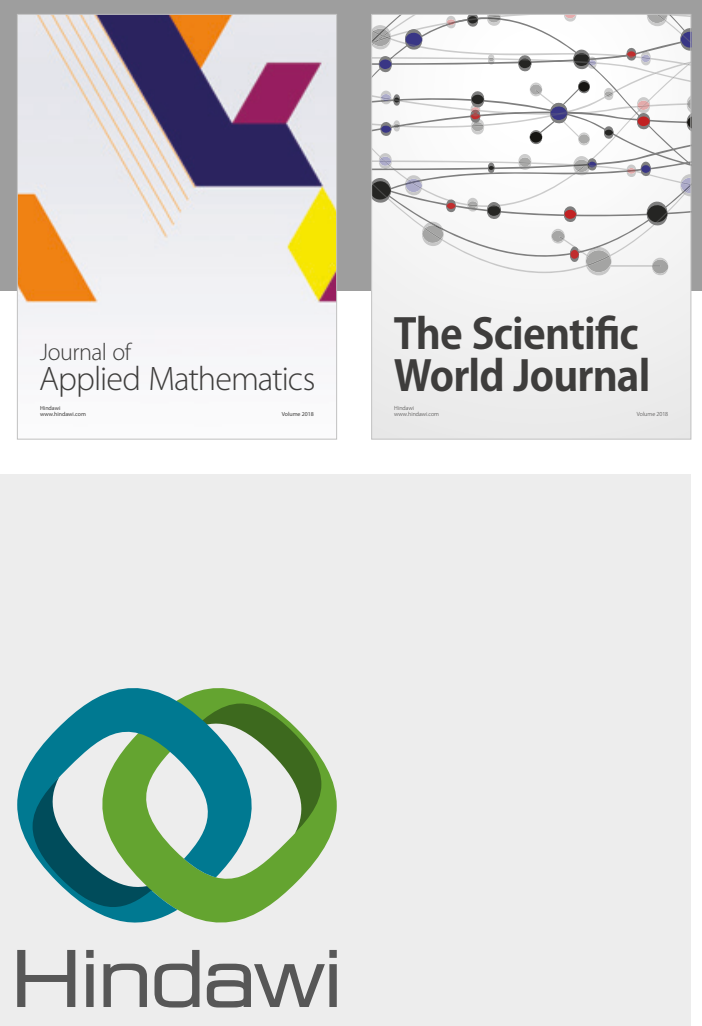

Submit your manuscripts at

www.hindawi.com

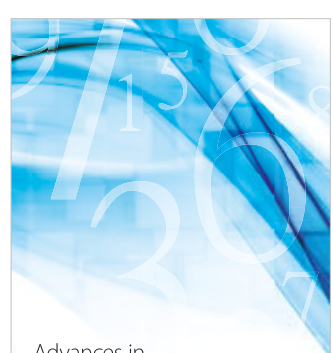

Advances in
Numerical Analysis
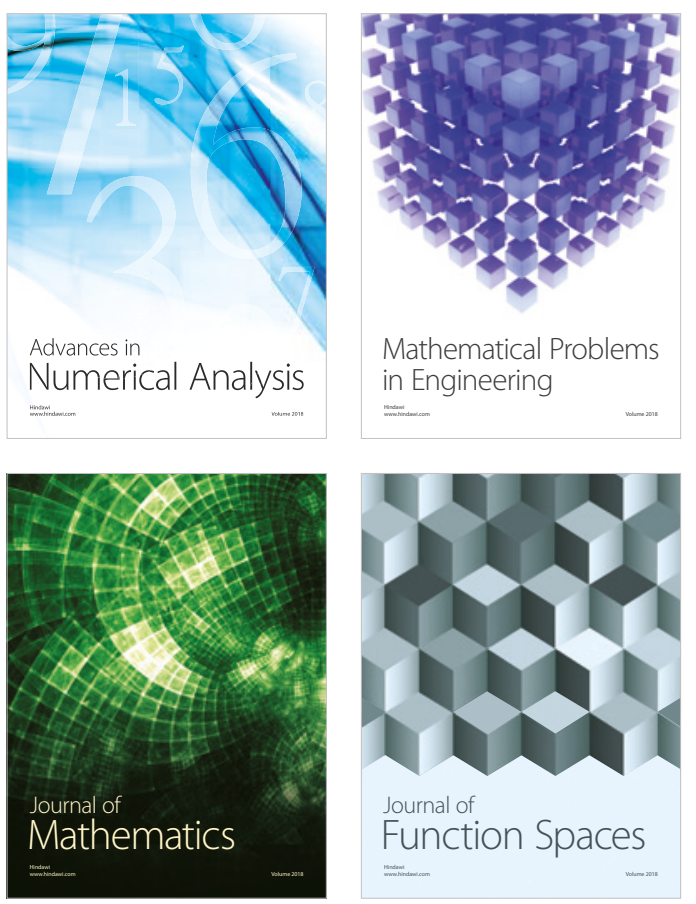

Mathematical Problems in Engineering

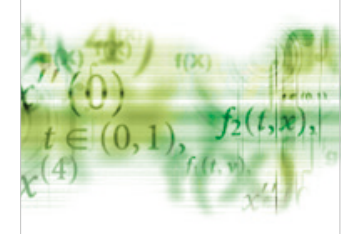

International Journal of

Differential Equations

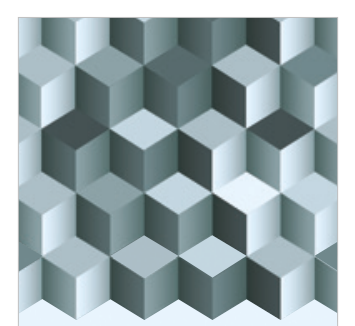

Journal of

Function Spaces

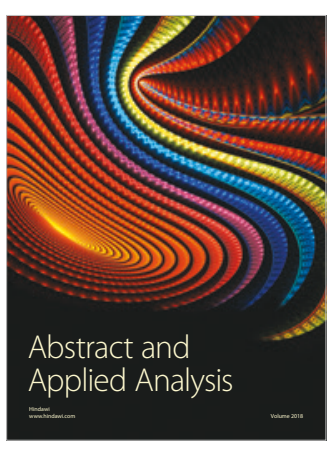

The Scientific

World Journal

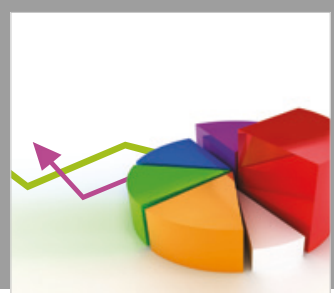

Journal of

Probability and Statistics
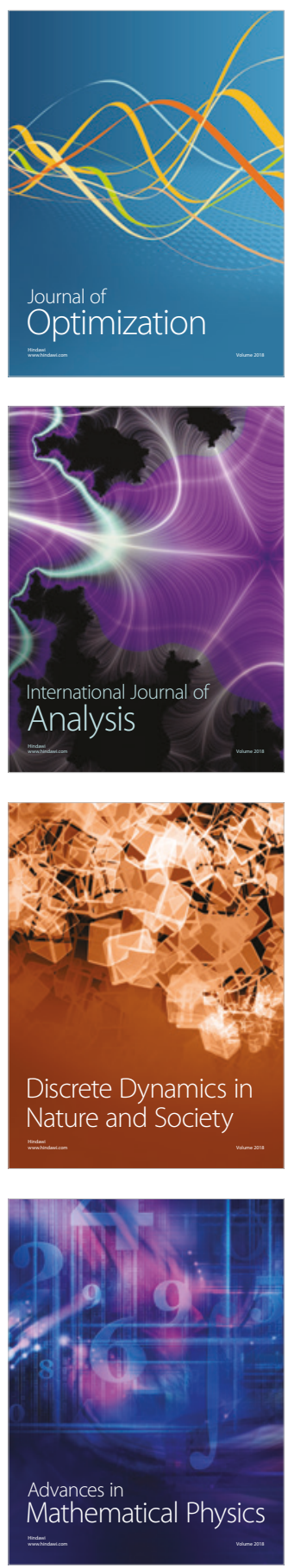